Pacific

Journal of

Mathematics

FREE RESOLUTIONS OF SOME SCHUBERT SINGULARITIES IN THE LAGRANGIAN GRASSMANNIAN

Venkatramani LaKshmibai And REUVEn Hodges

Volume $279 \quad$ No. 1-2

December 2015 


\title{
FREE RESOLUTIONS OF SOME SCHUBERT SINGULARITIES IN THE LAGRANGIAN GRASSMANNIAN
}

\author{
VENKATRAMANi LAKSHMiBAI AND REUVEN HODGES
}

Dedicated to the memory of Professor Robert Steinberg

\begin{abstract}
In this paper we construct free resolutions of a certain class of closed subvarieties of affine space of symmetric matrices (of a given size). Our class covers the symmetric determinantal varieties (i.e., determinantal varieties in the space of symmetric matrices), whose resolutions were first constructed by Józefiak, Pragacz and Weyman (1981). Our approach follows the techniques developed by Kummini, Lakshmibai, Pramathanath and Seshadri (2015), and uses the geometry of Schubert varieties.
\end{abstract}

\section{Introduction}

This paper is a sequel to [Kummini et al. 2015]. Lascoux [1978] constructed a minimal free resolution of the coordinate ring of the determinantal varieties (consisting of $m \times n$ matrices (over $\mathbb{C}$ ) of rank at most $k$, considered as a closed subvariety of the $m n$-dimensional affine space of all $m \times n$ matrices), as a module over the $m n$-dimensional polynomial ring (the coordinate ring of the $m n$-dimensional affine space).

In [Kummini et al. 2015], the authors construct free resolutions for a larger class of singularities, viz., Schubert singularities, i.e., the intersection of a singular Schubert variety and the "opposite big cell" inside a Grassmannian.

Józefiak, Pragacz and Weyman [1981] constructed a minimal free resolution of the coordinate ring of the determinantal varieties (in the space of symmetric matrices) as a module over the coordinate ring of the space of symmetric matrices. In this paper we construct free resolutions for a certain class of closed subvarieties of the affine space of symmetric matrices, which includes the symmetric determinantal varieties. The technique adopted in [Kummini et al. 2015] is algebraic group-theoretic, and we follow this approach.

The authors thank the referee for some useful comments; they also thank Manoj Kummini for helpful discussions. Lakshmibai was supported by NSA grant H98230-11-1-0197 and NSF grant 0652386. MSC2010: primary 20G20; secondary 14F05.

Keywords: Schubert varieties, Lagrangian Grassmannian, free resolutions. 
We now describe the results of this paper. Let $n$ be a positive integer. Let $V=\mathbb{C}^{2 n}$ and let $(\cdot, \cdot)$ be a nondegenerate skew-symmetric bilinear form on $V$. Let $H=\mathrm{SL}(V)$ and $G=\mathrm{SP}(V)=\{Z \in \mathrm{SL}(V) \mid Z$ leaves the form $(\cdot, \cdot)$ invariant $\}$. We take the matrix of the form, with respect to the standard basis of $V$, to be

$$
F=\left[\begin{array}{cc}
0 & J \\
-J & 0
\end{array}\right]
$$

where $J$ is the antidiagonal $(1, \ldots, 1)$, in this case of size $n$. To simplify our notation we will normally omit specifying the size of $J$ as it will be obvious from the context. We may realize $\operatorname{SP}(V)$ as the fixed point set of the involution $\sigma: H \rightarrow H$ given by $\sigma(Z)=F\left(Z^{T}\right)^{-1} F^{-1}$ (cf. [Steinberg 1968]).

Denoting by $T_{H}$ and $B_{H}$ the maximal torus in $H$ consisting of diagonal matrices and the Borel subgroup in $H$ consisting of upper triangular matrices, respectively, we have that $T_{H}$ and $B_{H}$ are stable under $\sigma$ and we set $T_{G}=T_{H}^{\sigma}, B_{G}=B_{H}^{\sigma}$. It is easily checked that $T_{G}$ is a maximal torus in $G$ and $B_{G}$ is a Borel subgroup in $G$.

Thus we obtain

$$
W_{G} \hookrightarrow W_{H}
$$

where $W_{G}, W_{H}$ denote the Weyl groups of $G, H$ respectively (with respect to $T_{G}, T_{H}$ respectively). Further, $\sigma$ induces an involution on $W_{H}$ :

$$
w=\left(a_{1}, \cdots, a_{2 n}\right) \in W_{H}, \quad \sigma(w)=\left(c_{1}, \cdots, c_{2 n}\right), \quad c_{i}=2 n+1-a_{2 n+1-i}
$$

and

$$
W_{G}=W_{H}^{\sigma} .
$$

Thus we obtain

$$
W_{G}=\left\{\left(a_{1} \cdots a_{2 n}\right) \in S_{2 n} \mid a_{i}=2 n+1-a_{2 n+1-i}, \quad 1 \leq i \leq 2 n\right\} .
$$

(here, $S_{2 n}$ is the symmetric group on $2 n$ letters). Thus $w=\left(a_{1} \cdots a_{2 n}\right) \in W_{G}$ is known once $\left(a_{1} \cdots a_{n}\right)$ is known. We shall denote an element $\left(a_{1} \cdots a_{2 n}\right)$ in $W_{G}$ by just $\left(a_{1} \cdots a_{n}\right)$. Further, for $w \in W_{G}$, denoting by $X_{G}(w)$ (resp. $\left.X_{H}(w)\right)$, the associated Schubert variety in $G / B_{G}$ (resp. $H / B_{H}$ ), we have that under the canonical inclusion $G / B_{G} \hookrightarrow H / B_{H}, X_{G}(w)=X_{H}(w) \cap G / B_{G}$, scheme-theoretically.

Let $P=P_{\hat{n}}$, the maximal parabolic subgroup of $G$ corresponding to omitting the simple root $\alpha_{n}$, the set of simple roots of $G$ being indexed as in [Bourbaki 1968]. Let $1 \leq k<r \leq n$ be positive integers, and let $w \in \mathcal{W}_{k, r}$ (cf. Notation 3.2). Our main result (cf. Theorem 3.22) is a description of the minimal free resolution of the coordinate ring of $Y_{P}(w):=X_{P}(w) \cap O_{G / P}^{-}$, the opposite cell of $X_{P}(w)$, as a module over the coordinate ring of $O_{G / P}^{-}$. For this, as in [Kummini et al. 2015], we use the Kempf-Lascoux-Weyman "geometric technique" of constructing minimal free resolutions; in fact we use the same notation and description of this technique as in [Kummini et al. 2015]. 
Suppose that we have a commutative diagram of varieties

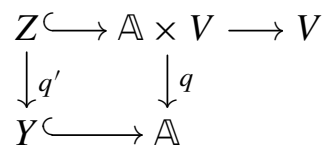

where $\mathbb{A}$ is an affine space, $Y$ a closed subvariety of $\mathbb{A}$ and $V$ a projective variety. The map $q$ is first projection, $q^{\prime}$ is proper and birational, and the inclusion $Z \hookrightarrow A \times V$ is a subbundle (over $V$ ) of the trivial bundle $\mathbb{A} \times V$. Let $\xi$ be the dual of the quotient bundle on $V$ corresponding to $Z$. Then the derived direct image $\mathbf{R} q_{*}^{\prime} \mathrm{O}_{Z}$ is quasi-isomorphic to a minimal complex $F$. with

$$
F_{i}=\bigoplus_{j \geq 0} H^{j}\left(V, \bigwedge^{i+j} \xi\right) \otimes_{\mathbb{C}} R(-i-j) .
$$

Here $R$ is the coordinate ring of $\mathbb{A}$; it is a polynomial ring and $R(k)$ refers to twisting with respect to its natural grading. If $q^{\prime}$ is such that the natural map $\mathrm{O}_{Y} \longrightarrow \mathbf{R} q_{*}^{\prime} \mathrm{O}_{Z}$ is a quasi-isomorphism (for example, if $q^{\prime}$ is a desingularization of $Y$ and $Y$ has rational singularities) then $F$. is a minimal free resolution of $\mathbb{C}[Y]$ over the polynomial ring $R$.

In applying this technique in any given situation, there are two main steps involved: one must find a suitable $Z$ and a suitable morphism $q^{\prime}: Z \longrightarrow Y$ such that the map $\mathrm{O}_{Y} \longrightarrow \mathbf{R} q_{*}^{\prime} \mathrm{O}_{Z}$ is a quasi-isomorphism and such that $Z$ is a vector bundle over a projective variety $V$; and, one must be able to compute the necessary cohomology groups. We carry this out for opposite cells $Y_{P}(w), w \in \mathcal{W}_{k, r}$.

As the first step, we establish the existence of a diagram as above, using the geometry of Schubert varieties. We now describe this briefly.

We take $\mathbb{A}=O_{G / P}^{-}$and $Y=Y_{P}(w)$. Let $\widetilde{P}$ be the two-step parabolic subgroup $P_{\widehat{r-k}, \hat{n}}$ of $G$, and let $\tilde{w}$ be the minimal representative of $w \widetilde{P}$ in $W^{\widetilde{P}}$ (that is, the set of minimal coset representatives in $W$, under the Bruhat order, of $W / W_{\widetilde{P}}$, where $W_{\widetilde{P}}$ is the Weyl group of $\widetilde{P})$. Let $w^{\prime}:=(k+1, \ldots, r, n, \ldots, r+1, k, \ldots, 1) \in S_{n}$, the Weyl group of $\mathrm{GL}_{n}$. Let $Z_{\widetilde{P}}(\tilde{w}):=Y_{P}(w) \times_{X_{P}(w)} X_{\widetilde{P}}(w)\left(=\left(O_{G / P}^{-} \times P / \widetilde{P}\right) \cap X_{\widetilde{P}}(w)\right)$. Then it turns out that $Z_{\widetilde{P}}(\tilde{w})$ is smooth (cf. Definition 3.20), and is a desingularization of $Y_{P}(w)$. Write $p$ for the composite map $Z_{\widetilde{P}}(\tilde{w}) \hookrightarrow O_{G / P}^{-} \times P / \widetilde{P} \rightarrow P / \widetilde{P}$ where the first map is the inclusion and the second map is the projection. We have (cf. Theorem 3.22) that $p$ identifies $Z_{\widetilde{P}}(\tilde{w})$ as a subbundle of the trivial bundle $O_{G / P}^{-} \times X_{P_{r-k}^{\prime}}\left(w^{\prime}\right)$ over $X_{P_{r-k}^{\prime}}\left(w^{\prime}\right)$, which arises as the restriction (to $X_{P^{\prime}}\left(w^{\prime}\right)$ ) of a certain homogeneous vector bundle on $\mathrm{GL}_{n} / P_{r-k}^{\prime}$. With $V:=X_{P_{r-k}^{\prime}}\left(w^{r-k}\right)^{r-k}$, we get:

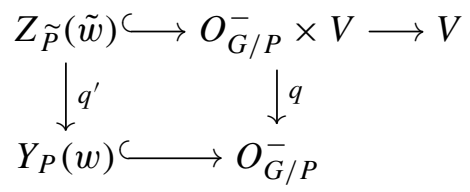


In this diagram, $q^{\prime}$ is a desingularization of $Y_{P}(w)$. Since it is known that Schubert varieties have rational singularities, we have that the map $\mathrm{O}_{Y} \longrightarrow \mathbf{R} q_{*}^{\prime} \mathrm{O}_{Z}$ is a quasi-isomorphism, so $F$. is a minimal resolution.

At the second step, we need to determine the cohomology of the bundles $\wedge^{t} \xi$ over $V$. In the above situation, $V=X_{P_{r-k}^{\prime}}\left(w^{\prime}\right) \hookrightarrow \mathrm{GL}_{n} / P_{r-k}^{\prime}$. As can be easily seen, $X_{P_{r-k}^{\prime}}\left(w^{\prime}\right)$ is a Grassmannian, namely, ${ }^{r-k} L_{r} / P_{r-k}^{\prime \prime}$; the bundles $\bigwedge^{t} \xi$ (on $\mathrm{GL}_{r} / P_{r-k}^{\prime \prime}$ ) are also homogeneous, but are not of Bott type: they are not completely reducible (so one can not apply the Bott algorithm for computing the cohomology). This can be resolved in two ways. In [Ottaviani and Rubei 2006] the authors determine the cohomology of general homogeneous bundles on Hermitian symmetric spaces, and thus their results can be used to determine $H^{\bullet}\left(V, \bigwedge^{t} \xi\right)$. Alternatively, using a technique from [Weyman 2003], we may compute the resolution of a related space (whose associated homogeneous vector bundle is of Bott type) from which we retrieve the resolution of the coordinate ring of $Y_{P}(w)$ as a subcomplex.

We hope to extend the results of this paper to Schubert varieties in the orthogonal Grassmannian. Details will appear in a subsequent paper.

The paper is organized as follows. Section 2 contains notations and conventions and the necessary background material on Schubert varieties in the flag variety (Section 2.1) and Schubert varieties in the symplectic flag variety (Sections 2.2 and 2.3) and homogeneous bundles (Section 2.4). In Section 3, we discuss properties of Schubert desingularization, including the construction of Diagram 1.2. Section 4 is devoted to a review of the Kempf-Lascoux-Weyman technique and completes step one of the two part process of the geometric technique. Section 5 explains how the cohomology groups of the homogeneous bundles constructed in step one may be calculated.

\section{Preliminaries}

In this section we collect various results about Schubert varieties in the flag variety and symplectic flag variety, homogeneous vector bundles, and the Bott algorithm.

2.1. Notation and conventions in type A. We collect the symbols used and the conventions adopted in the rest of the paper here. For details on algebraic groups and Schubert varieties, the reader may refer to [Borel 1991; Jantzen 2003; Billey and Lakshmibai 2000; Seshadri 2007].

Let $N$ be positive integer. We denote by $\mathrm{GL}_{N}$ (respectively, $B_{N}, B_{N}^{-}$) the group of all (respectively, upper triangular, lower triangular) invertible $N \times N$ matrices over $\mathbb{C}$. The Weyl group $W$ of $\mathrm{GL}_{N}$ is isomorphic to the group $S_{N}$ of permutations of $N$ symbols and is generated by the simple reflections $s_{i}$, which correspond to the transpositions $(i, i+1)$, for $1 \leq i \leq N-1$. For $w \in W$, its length is the smallest integer $l$ such that $w=s_{i_{1}} \cdots s_{i_{l}}$ as a product of simple reflections. For every 
$1 \leq i \leq N-1$, there is a minimal parabolic subgroup $P_{i}$ containing $s_{i}$ (thought of as an element of $\mathrm{GL}_{N}$ ) and a maximal parabolic subgroup $P_{\hat{i}}$ not containing $s_{i}$. Any parabolic subgroup can be written as $P_{\widehat{A}}:=\bigcap_{i \in A} P_{\hat{i}}$ for some $A \subset\{1, \ldots, N-1\}$. On the other hand, for $A \subseteq\{1, \ldots, N-1\}$ write $P_{A}$ for the subgroup of $\mathrm{GL}_{N}$ generated by $P_{i}$ for $i \in A$. Then $P_{A}$ is a parabolic subgroup and $P_{\{1, \ldots, N-1\} \backslash A}=P_{\widehat{A}}$.

We write the elements of $W$ in one-line notation: $\left(a_{1}, \ldots, a_{N}\right)$ is the permutation $i \mapsto a_{i}$. For any $A \subseteq\{1, \ldots, N-1\}$, define $W_{P_{A}}$ to be the subgroup of $W$ generated by $\left\{s_{i}: i \in A\right\}$. By $W^{P_{A}}$ we mean the subset of $W$ consisting of the minimal representatives (under the Bruhat order) in $W$ of the elements of $W / W_{P_{A}}$. For $1 \leq i \leq N$, we represent the elements of $W^{P_{\hat{i}}}$ by sequences $\left(a_{1}, \ldots, a_{i}\right)$ with $1 \leq a_{1}<\cdots<a_{i} \leq N$ since under the action of the group $W_{P_{\hat{i}}}$, every element of $W$ can be represented minimally by such a sequence.

We identify $\mathrm{GL}_{N}=\mathrm{GL}(V)$ for some $N$-dimensional vector-space $V$. Let $A:=$ $\left\{i_{1}<i_{2}<\cdots<i_{r}\right\} \subseteq\{1, \ldots, N-1\}$. Then $\mathrm{GL}_{N} / P_{\widehat{A}}$ is the set of all flags $0=V_{0} \subsetneq V_{1} \subsetneq V_{2} \subsetneq \cdots \subsetneq V_{r} \subsetneq V$ of subspaces $V_{j}$ of dimension $i_{j}$ inside $V$. We call $\mathrm{GL}_{N} / P_{\widehat{A}}$ a flag variety. If $A=\{1, \ldots, N-1\}$ (i.e., $P_{\widehat{A}}=B_{N}$ ), then we call the flag variety a full flag variety; otherwise, a partial flag variety. The Grassmannian Grass $_{i, N}$ of $i$-dimensional subspaces of $V$ is $\mathrm{GL}_{N} / P_{\hat{i}}$.

Let $\widetilde{P}$ be any parabolic subgroup containing $B_{N}$ and $\tau \in W$. The Schubert variety $X_{\widetilde{P}}(\tau)$ is the closure inside $\mathrm{GL}_{N} / \widetilde{P}$ of $B_{N} \cdot e_{w}$ where $e_{w}$ is the coset $\tau \widetilde{P}$, endowed with the canonical reduced scheme structure. Hereafter, when we write $X_{\widetilde{P}}(\tau)$, we mean that $\tau$ is the representative in $W^{\widetilde{P}}$ of its coset. The opposite big cell $O_{\mathrm{GL}_{N} / \widetilde{P}}^{-}$in $\mathrm{GL}_{N} / \widetilde{P}$ is the $B_{N}^{-}$-orbit of the coset (id $\cdot \widetilde{P}$ ) in $\mathrm{GL}_{N} / \widetilde{P}$. Let $Y_{\widetilde{P}}(\tau):=X_{\widetilde{P}}(\tau) \cap O_{\mathrm{GL}_{N} / \widetilde{P}}^{-}$; we refer to $Y_{\widetilde{P}}(\tau)$ as the opposite cell of $X_{\widetilde{P}}(\tau)$.

We will write $R^{+}, R_{\widetilde{P}}^{-}, R_{\widetilde{P}}^{+}, R_{\widetilde{P}}^{-}$, to denote respectively, positive and negative roots for $\mathrm{GL}_{N}$ and for $\widetilde{P}$. We denote by $\epsilon_{i}$ the character that sends the invertible diagonal matrix with $t_{1}, \ldots, t_{n}$ on the diagonal to $t_{i}$.

2.2. Notation and conventions in type $\boldsymbol{C}$. Below we review the properties of symplectic Schubert varieties relevant to this paper. For a more in-depth introduction the reader may refer to [Lakshmibai and Raghavan 2008, Chapter 6].

Let $n$ be a positive integer. Let $V=\mathbb{C}^{2 n}$ and let $(\cdot, \cdot)$ be a nondegenerate skew-symmetric bilinear form on $V$. Let $H=\operatorname{SL}(V)$ and $G=\operatorname{SP}(V)=$ $\{Z \in \mathrm{SL}(V) \mid Z$ leaves the form $(\cdot, \cdot)$ invariant $\}$. We take the matrix of the form, with respect to the standard basis of $V$, to be

$$
F=\left[\begin{array}{cc}
0 & J \\
-J & 0
\end{array}\right]
$$

where $J$ is the antidiagonal $(1, \ldots, 1)$, in this case of size $n$. To simplify our notation we will normally omit specifying the size of $J$ as it will be obvious from the context. 
We may realize $\operatorname{SP}(V)$ as the fixed point set of the involution $\sigma: H \rightarrow H$ given by $\sigma(Z)=F\left(Z^{T}\right)^{-1} F^{-1}$ (cf. [Steinberg 1968]). That is,

$$
\begin{aligned}
G & =\left\{Z \in \mathrm{SL}(V) \mid Z^{T} F Z=F\right\} \\
& =\left\{Z \in \mathrm{SL}(V) \mid F^{-1}\left(Z^{T}\right)^{-1} F=Z\right\} \\
& =\left\{Z \in \mathrm{SL}(V) \mid F\left(Z^{T}\right)^{-1} F^{-1}=Z\right\} \\
& =H^{\sigma} .
\end{aligned}
$$

Denote by $T_{H}$ and $B_{H}$ the maximal torus in $H$ consisting of diagonal matrices and the Borel subgroup in $H$ consisting of upper triangular matrices, respectively. It is easily seen that $T_{H}$ and $B_{H}$ are stable under $\sigma$ and we set $T_{G}=T_{H}^{\sigma}, B_{G}=B_{H}^{\sigma}$. It is easily checked that $T_{G}$ is a maximal torus in $G$ and $B_{G}$ is a Borel subgroup in $G$.

Thus we obtain

$$
W_{G} \hookrightarrow W_{H}
$$

where $W_{G}, W_{H}$ denote the Weyl groups of $G, H$, respectively (with respect to $T_{G}, T_{H}$, respectively). Further, $\sigma$ induces an involution on $W_{H}$ :

$$
w=\left(a_{1}, \cdots, a_{2 n}\right) \in W_{H}, \quad \sigma(w)=\left(c_{1}, \cdots, c_{2 n}\right), \quad c_{i}=2 n+1-a_{2 n+1-i}
$$

and

$$
W_{G}=W_{H}^{\sigma}
$$

Thus we obtain

$$
W_{G}=\left\{\left(a_{1} \cdots a_{2 n}\right) \in S_{2 n} \mid a_{i}=2 n+1-a_{2 n+1-i}, \quad 1 \leq i \leq 2 n\right\} .
$$

(here, $S_{2 n}$ is the symmetric group on $2 n$ letters). Thus $w=\left(a_{1} \cdots a_{2 n}\right) \in W_{G}$ is known once $\left(a_{1} \cdots a_{n}\right)$ is known. We shall denote an element $\left(a_{1} \cdots a_{2 n}\right)$ in $W_{G}$ by just $\left(a_{1} \cdots a_{n}\right)$. For example, $(4231) \in S_{4}$ represents $(42) \in W_{G}, G=\operatorname{SP}(4)$.

The involution $\sigma$ induces an involution on $X\left(T_{H}\right)$, the character group of $T_{H}$ :

$$
\chi \in X\left(T_{H}\right), \quad \sigma(\chi)(D)=\chi(\sigma(D)), \quad D \in T_{H} .
$$

Let $\epsilon_{i}$, for $1 \leq i \leq 2 n$, be the character in $X\left(T_{H}\right), \epsilon_{i}(D)=d_{i}$, the $i$-th entry in $D \in T_{H}$. We have

$$
\sigma\left(\epsilon_{i}\right)=-\epsilon_{2 n+1-i}
$$

Now it is easily seen that the under the canonical surjective map

$$
\varphi: X\left(T_{H}\right) \rightarrow X\left(T_{G}\right)
$$

we have

$$
\varphi\left(\epsilon_{i}\right)=-\varphi\left(\epsilon_{2 n+1-i}\right), \quad 1 \leq i \leq 2 n .
$$


Let $R_{H}:=\left\{\epsilon_{i}-\epsilon_{j}, 1 \leq i, j \leq 2 n\right\}$ be the root system of $H$ (relative to $T_{H}$ ), and $R_{H}^{+}:=\left\{\epsilon_{i}-\epsilon_{j}, 1 \leq i<j \leq 2 n\right\}$ the set of positive roots (relative to $B_{H}$ ). We have the following:

(a) $\sigma$ leaves $R_{H}$ (resp. $R_{H}^{+}$) stable.

(b) For $\alpha, \beta \in R_{H}, \varphi(\alpha)=\varphi(\beta) \Leftrightarrow \alpha=\sigma(\beta)$.

(c) $\varphi$ is equivariant for the canonical action of $W_{G}$ on $X\left(T_{H}\right), X\left(T_{G}\right)$.

(d) $R_{H}^{\sigma}=\left\{ \pm\left(\epsilon_{i}-\epsilon_{2 n+1-i}\right), 1 \leq i \leq n\right\}$.

Let $R_{G}$ (resp. $R_{G}^{+}$) be the set of roots of $G$ with respect to $T_{G}$ (resp. the set of positive roots with respect to $B_{G}$ ). Using the above facts and the explicit nature of the adjoint representation of $G$ on Lie $G$, we deduce that

$$
R_{G}=\varphi\left(R_{H}\right), \quad R_{G}^{+}=\varphi\left(R_{H}^{+}\right) .
$$

In particular, $R_{G}$ (resp. $R_{G}^{+}$) gets identified with the orbit space of $R_{H}\left(\right.$ resp. $R_{H}^{+}$) modulo the action of $\sigma$. Thus we obtain the following identification:

$$
\begin{gathered}
R_{G}=\left\{ \pm\left(\epsilon_{i} \pm \epsilon_{j}\right), 1 \leq i<j \leq n\right\} \cup\left\{ \pm 2 \epsilon_{i}, i=1, \ldots, n\right\}, \\
R_{G}^{+}=\left\{\left(\epsilon_{i} \pm \epsilon_{j}\right), 1 \leq i<j \leq n\right\} \cup\left\{2 \epsilon_{i}, i=1, \ldots, n\right\} .
\end{gathered}
$$

The set $S_{G}$ of simple roots in $R_{G}^{+}$is given by

$$
S_{G}:=\left\{\alpha_{i}=\epsilon_{i}-\epsilon_{i+1}, 1 \leq i \leq n-1\right\} \cup\left\{\alpha_{n}=2 \epsilon_{n}\right\} .
$$

Let us denote the simple reflections in $W_{G}$ by $\left\{s_{i}, 1 \leq i \leq n\right\}$, namely, $s_{i}=$ reflection with respect to $\epsilon_{i}-\epsilon_{i+1}$ for $1 \leq i \leq n-1$, and $s_{n}=$ reflection with respect to $2 \epsilon_{n}$. Then we have

$$
s_{i}= \begin{cases}r_{i} r_{2 n-i}, & \text { if } 1 \leq i \leq n-1, \\ r_{n}, & \text { if } i=n,\end{cases}
$$

where $r_{i}$ denotes the transposition $(i, i+1)$ in $S_{2 n}$ for $1 \leq i \leq 2 n-1$.

For $w \in W_{G}$, let us denote by $l\left(w, W_{H}\right)\left(\right.$ resp. $\left.l\left(w, W_{G}\right)\right)$ the length of $w$ as an element of $W_{H}$ (resp. $\left.W_{G}\right)$. For $w=\left(a_{1}, \cdots, a_{2 n}\right) \in W_{H}$, denote

$$
m(w):=\#\left\{i \leq n \mid a_{i}>n\right\} .
$$

Then for $w=\left(a_{1}, \cdots, a_{2 n}\right) \in W_{G}$, we have $l\left(w, W_{G}\right)=\frac{1}{2}\left(l\left(w, W_{H}\right)+m(w)\right)$.

Proposition 2.2.3 [Lakshmibai and Raghavan 2008, Proposition 6.2.5.1]. Let $w \in W_{G}$; let $X_{G}(w)$ (resp. $X_{H}(w)$ ) be the associated Schubert variety in $G / B_{G}$ (resp. $\left.H / B_{H}\right)$. Under the canonical inclusion $G / B_{G} \hookrightarrow H / B_{H}$, we have $X_{G}(w)=$ $X_{H}(w) \cap G / B_{G}$. Further, the intersection is scheme-theoretic. 
Notation 2.2.4. For the remainder of the paper we fix the following notation. Let $1 \leq k<r \leq n$ be positive integers. Let $Q=Q_{\hat{n}}$ to be the parabolic subgroup of $H$ corresponding to omitting the root $\alpha_{n}$ and $P=P_{\hat{n}}$ to be the parabolic subgroup of $G$ corresponding to omitting the root $\alpha_{n}$. Let $\widetilde{P}$ be the two-step parabolic subgroup $P_{\widehat{r-k}, \hat{n}}$ of $G$. Let $\widetilde{Q}$ be the three step parabolic subgroup $Q_{\widehat{\widetilde{r-k}}, \hat{n}, 2 n-(r-k)}$ in $H$. Note that $P=Q^{\sigma}$ and $\widetilde{P}=\widetilde{Q}^{\sigma}$. Finally, we identify $P / \widetilde{P}$ with $\mathrm{GL}_{n} / P_{r-k}^{\prime}$ where $P_{r-k}^{\prime}$ is the parabolic subgroup of $\mathrm{GL}_{n}$ corresponding to omitting the $\operatorname{root} \alpha_{r-k}$.

Definition 2.2.5. A square $m \times m$ matrix $X$ is persymmetric if $J X=X^{T} J$. Or, equivalently, if $J X$ is symmetric.

Remark 2.2.6. We denote by $\mathrm{Mat}_{n}$ the space of $n \times n$ matrices. Let $K$ be the subgroup of $H$ consisting of matrices of the form

$$
\left[\begin{array}{cc}
\operatorname{Id}_{n} & 0 \\
Y & \mathrm{Id}_{n}
\end{array}\right], \quad Y \in \mathrm{Mat}_{n} .
$$

The canonical morphism $H \rightarrow H / Q$ induces a morphism $\psi_{H}: K \rightarrow H / Q$. We have that $\psi_{H}$ is an open immersion, and $\psi_{H}(K)$ gets identified with the opposite big cell $O_{H / Q}^{-}$in $H / Q$.

The cell $O_{H / Q}^{-}$is $\sigma$-stable and by [Lakshmibai and Raghavan 2008, Corollary 6.2.4.3], we can identify the opposite big cell $O_{G / P}^{-}$as

$$
O_{G / P}^{-}=\left(O_{H / Q}^{-}\right)^{\sigma}=\left\{z \in K \mid J Y^{T} J=Y\right\} .
$$

So $O_{G / P}^{-}$is the subspace of $K$ with $Y$ persymmetric. Thus we can identify $O_{G / P}^{-}$ with the space of symmetric $n \times n$ matrices, $\operatorname{Sym}_{n}$, under the map $O_{G / P}^{-} \longrightarrow \operatorname{Sym}_{n}$ given by

$$
\left[\begin{array}{cc}
\operatorname{Id}_{n} & 0 \\
Y & \operatorname{Id}_{n}
\end{array}\right] \mapsto J Y
$$

2.3. Opposite cells in Schubert varieties in the symplectic flag variety. A matrix $z \in \operatorname{SL}(V)$ with $n \times n$ block form

$$
\left[\begin{array}{cc}
A_{n \times n} & C_{n \times n} \\
D_{n \times n} & E_{n \times n}
\end{array}\right]
$$

is an element of $G$ if and only if $z^{T} F z=F$, i.e., if and only if the following conditions hold on the $n \times n$ blocks:

$$
\begin{aligned}
A^{T} J D & =D^{T} J A, \\
C^{T} J E & =E^{T} J C, \\
J=\left(A^{T} J E-D^{T} J C\right) & =\left(E^{T} J A-C^{T} J D\right) .
\end{aligned}
$$

The following proposition will prove useful throughout the rest of the paper. 
Proposition 2.3.4. Write $U_{P}^{-}$for the negative unipotent radical of $P$.

(a) $O_{G / P}^{-}$can be naturally identified with $U_{P}^{-} P / P$

(b) For

$$
z=\left[\begin{array}{cc}
A_{n \times n} & C_{n \times n} \\
D_{n \times n} & E_{n \times n}
\end{array}\right] \in G
$$

$z P \in O_{G / P}^{-}$if and only if $A$ is invertible.

(c) The inverse image of $O_{G / P}^{-}$under the natural map $G / \widetilde{P} \rightarrow G / P$ is isomorphic to $O_{G / P}^{-} \times P / \widetilde{P}$ as schemes. Every element of $O_{G / P}^{-} \times P / \widetilde{P}$ is of the form

$$
\left[\begin{array}{cc}
A_{n \times n} & 0 \\
D_{n \times n} & J\left(A^{T}\right)^{-1} J
\end{array}\right] \bmod \widetilde{P} \in G / \widetilde{P} .
$$

Moreover, two matrices

$$
\left[\begin{array}{cc}
A_{n \times n} & 0_{n \times n} \\
D_{n \times n} & J\left(A^{T}\right)^{-1} J
\end{array}\right] \text { and }\left[\begin{array}{cc}
A_{n \times n}^{\prime} & 0_{n \times n} \\
D_{n \times n}^{\prime} & J\left(A^{\prime T}\right)^{-1} J
\end{array}\right]
$$

in $G$ represent the same element modulo $\widetilde{P}$ if and only if there exists a matrix $q \in P_{\widehat{r-k}}^{\prime}$ (as defined in Notation 2.2.4) such that $A^{\prime}=A q$ and $D^{\prime}=D q$.

(d) $P / \widetilde{P}$ is isomorphic to $\mathrm{GL}_{n} / P_{r-k}^{\prime}$. In particular, the projection map $O_{G / P}^{-} \times$ $P / \widetilde{P} \rightarrow P / \widetilde{P}$ is given by

$$
\left[\begin{array}{cc}
A_{n \times n} & 0 \\
D_{n \times n} & J\left(A^{T}\right)^{-1} J
\end{array}\right] \bmod \widetilde{P} \longmapsto A \bmod P_{r-k}^{\prime} \in \mathrm{GL}_{n} / P_{r-k}^{\prime} \cong P / \widetilde{P} .
$$

Proof. (a): Note that $U_{P}^{-}$is the subgroup of $G$ generated by the root subgroups $U_{-\alpha}$ for $\alpha \in R^{+} \backslash R_{P}^{+}$. Under the canonical projection $G \rightarrow G / P, g \mapsto g P, U_{P}^{-}$ is mapped isomorphically onto its image $O_{G / P}^{-}$(cf. [Billey and Lakshmibai 2000, Section 4.4.4]). Thus we obtain the identification of $O_{G / P}^{-}$with $U_{P}^{-} P / P$.

(b): Suppose that $z P \in O_{G / P}^{-}$. By (a) this means that $\exists n \times n$ matrices $A^{\prime}, C^{\prime}, D^{\prime}, E^{\prime}$ such that

$z_{1}=\left[\begin{array}{cc}\mathrm{Id}_{n} & 0 \\ D^{\prime} & \mathrm{Id}_{n}\end{array}\right] \in U_{P}^{-} \quad$ and $\quad z_{2}=\left[\begin{array}{cc}A^{\prime} & C^{\prime} \\ 0 & E^{\prime}\end{array}\right] \in P \quad$ with $\quad z=\left[\begin{array}{cc}A & C \\ D & E\end{array}\right]=z_{1} z_{2}$.

Hence $A=A^{\prime}$, and $A^{\prime}$ invertible implies $A$ invertible.

Conversely, suppose $A$ is invertible. Let

$$
z=\left[\begin{array}{ll}
A & C \\
D & E
\end{array}\right] \in G
$$


Then $A, C, D, E$ satisfy properties (2.3.1)-(2.3.2). Since $A$ is invertible we may write

$$
z=z_{1} z_{2} \quad \text { where } \quad z_{1}=\left[\begin{array}{cc}
\mathrm{Id}_{n} & 0 \\
D A^{-1} & \mathrm{Id}_{n}
\end{array}\right], z_{2}=\left[\begin{array}{cc}
A & C \\
0 & E-D A^{-1} C
\end{array}\right]
$$

We shall now show that $z_{1}, z_{2} \in G$. First, we note that (2.3.1) implies that

$$
J\left(D A^{-1}\right)=\left(D A^{-1}\right)^{T} J .
$$

Then (2.3.5) shows that $z_{1} \in U_{P}^{-}$, and hence $z_{1} \in G$.

Now $z_{1} \in G$ implies $z_{1}^{-1} \in G$, and $z \in G$ by assumption. Hence $z_{2}=z z_{1}^{-1} \in G$. Further, since $A$ is invertible, $z_{2} \in P$. Hence the coset $z P=z_{1} P$, which in view of the fact that $z_{1} \in U_{P}^{-}$, implies by part (a) that $z P \in O_{G / P}^{-}$.

(c): Let $z \in U_{P}^{-} P \subset G$. Then we can write $z=z_{1} z_{2}$ uniquely with $z_{1} \in U_{P}^{-}, z_{2} \in P$. Suppose that

$$
\left[\begin{array}{cc}
\mathrm{Id}_{n} & 0 \\
D_{n \times n} & \mathrm{Id}_{n}
\end{array}\right]\left[\begin{array}{cc}
A_{n \times n} & C_{n \times n} \\
0_{n \times n} & E_{n \times n}
\end{array}\right]=\left[\begin{array}{cc}
\mathrm{Id}_{n} & 0 \\
D_{n \times n}^{\prime} & \operatorname{Id}_{n}
\end{array}\right]\left[\begin{array}{cc}
A_{n \times n}^{\prime} & C_{n \times n}^{\prime} \\
0_{n \times n} & E_{n \times n}^{\prime}
\end{array}\right]
$$

then $A=A^{\prime}, C=C^{\prime}, D A=D^{\prime} A^{\prime}$ and $D C+E=D^{\prime} C^{\prime}+E^{\prime}$, which yields that $D^{\prime}=D$ (since $A=A^{\prime}$ is invertible), and then $E=E^{\prime}$. Hence $U_{P}^{-} \times_{\mathbb{C}} P=U_{P}^{-} P$. Thus for any parabolic subgroup $P^{\prime} \subseteq P, U_{P}^{-} \times_{\mathbb{C}} P / P^{\prime}=U_{P}^{-} P / P^{\prime}$. The asserted isomorphism follows by part (a) from taking $P^{\prime}=\widetilde{P}$.

To see the second assertion consider

$$
z=\left[\begin{array}{cc}
A_{n \times n} & C_{n \times n} \\
D_{n \times n} & E_{n \times n}
\end{array}\right] \in G
$$

with $z P \in O_{G / P}^{-}$. Note that the $n \times n$ block matrices satisfy properties (2.3.1)-(2.3.3) and by (b), $A$ is invertible.

We have by the first part of (c) that the coset $z P$ is an element of $O_{G / P}^{-} \times P / \widetilde{P}$, since $z P \in O_{G / P}^{-}$.

Claim. We have a decomposition of $z$ in $G$,

$$
\left[\begin{array}{ll}
A & C \\
D & E
\end{array}\right]=y_{1} y_{2} \text { where } y_{1}=\left[\begin{array}{cc}
A & 0 \\
D & J\left(A^{T}\right)^{-1} J
\end{array}\right] \in G, y_{2}=\left[\begin{array}{cc}
\operatorname{Id}_{n} & A^{-1} C \\
0 & \operatorname{Id}_{n}
\end{array}\right] \in \widetilde{P} \text {. }
$$

We first check that $z=y_{1} y_{2}$. We need the following identity

$$
J A^{T} J\left(E-D A^{-1} C\right)=\operatorname{Id}_{n},
$$

which follows from

$$
\begin{aligned}
J A^{T} J\left(E-D A^{-1} C\right) & =J\left(A^{T} J E-A^{T} J D A^{-1} C\right) \\
& =J\left(A^{T} J E-D^{T} J A A^{-1} C\right) \\
& =J J \\
& =\operatorname{Id}_{n} .
\end{aligned}
$$


So that

$$
\begin{aligned}
D A^{-1} C+J\left(A^{T}\right)^{-1} J & =D A^{-1} C+J\left(A^{T}\right)^{-1} J J A^{T} J\left(E-D A^{-1} C\right) \\
& =D A^{-1} C+E-D A^{-1} C \\
& =E .
\end{aligned}
$$

With this it is easily verified that $z=y_{1} y_{2}$.

It is clear that $y_{1} \in G$. To show $y_{2} \in G$ we need to check that $J\left(A^{-1} C\right)^{T} J=A^{-1} C$.

$$
\begin{aligned}
\left(A^{-1} C\right)^{T} J & =\left(A^{-1} C\right)^{T} J J A^{T} J\left(E-D A^{-1} C\right) \\
& =C^{T} J\left(E-D A^{-1} C\right) \\
& =E^{T} J C-C^{T} J D A^{-1} C \\
& =\left(E-D A^{-1} C\right)^{T} J C \\
& =\left(E-D A^{-1} C\right)^{T} J A J J A^{-1} C \\
& =\left(J A^{T} J\left(E-D A^{-1} C\right)\right)^{T} J\left(A^{-1} C\right) \\
& =J\left(A^{-1} C\right)
\end{aligned}
$$

Thus $y_{2} \in G$. It is clear additionally that $y_{2} \in \widetilde{P}$ (in fact $y_{2} \in B_{G}$ ).

Hence our claim follows and we have

$$
\left[\begin{array}{ll}
A & C \\
D & E
\end{array}\right]=\left[\begin{array}{cc}
A & 0 \\
D & J\left(A^{T}\right)^{-1} J
\end{array}\right] \bmod \widetilde{P}
$$

Finally,

$$
\left[\begin{array}{cc}
A_{n \times n} & 0_{n \times n} \\
D_{n \times n} & J\left(A^{T}\right)^{-1} J
\end{array}\right]=\left[\begin{array}{cc}
A_{n \times n}^{\prime} & 0_{n \times n} \\
D_{n \times n}^{\prime} & J\left(A^{\prime T}\right)^{-1} J
\end{array}\right] \bmod \widetilde{P}
$$

if and only if there exist matrices $q \in P_{r-k}^{\prime}$, and $q^{\prime} \in \mathrm{Mat}_{n}$ such that

$$
\left[\begin{array}{cc}
A^{\prime} & 0_{n \times n} \\
D^{\prime} & J\left(A^{T}\right)^{-1} J
\end{array}\right]=\left[\begin{array}{cc}
A & 0_{n \times n} \\
D & J\left(A^{\prime T}\right)^{-1} J
\end{array}\right]\left[\begin{array}{cc}
q & q^{\prime} \\
0_{n \times n} & J\left(q^{T}\right)^{-1} J
\end{array}\right],
$$

which holds if and only if $q^{\prime}=0, A^{\prime}=A q$ and $D^{\prime}=D q$ (since $A$ and $A^{\prime}$ are invertible).

(d): There is a surjective morphism of $\mathbb{C}$-group schemes $P \rightarrow \mathrm{GL}_{n}$ :

$$
\left[\begin{array}{cc}
A & C \\
0 & E
\end{array}\right] \rightarrow A
$$

This induces the required isomorphism. The element

$$
\left[\begin{array}{ll}
A & C \\
D & E
\end{array}\right] \bmod \widetilde{P} \in O_{G / P}^{-} \times P / \widetilde{P}
$$

decomposes uniquely as

$$
\left[\begin{array}{cc}
\mathrm{Id}_{n} & 0 \\
D A^{-1} & \mathrm{Id}_{n}
\end{array}\right]\left(\left[\begin{array}{cc}
A & C \\
0 & E-D A^{-1} C
\end{array}\right] \bmod \widetilde{P}\right)
$$

and hence it is mapped to $A \bmod P_{r-k}^{\prime}$. 
2.4. Homogeneous bundles and representations. Let $Q$ be a parabolic subgroup of $\mathrm{GL}_{n}$. We collect here some results about homogeneous vector bundles on $\mathrm{GL}_{n} / Q$. Most of these results are well-known, but for some of them, we could not find a reference, so we give a proof here for the sake of completeness. Online notes of G. Ottaviani [1995] and of D. Snow [1994] discuss the details of many of these results.

Let $L_{Q}$ and $U_{Q}$ be respectively the Levi subgroup and the unipotent radical of $Q$. Let $E$ be a finite-dimensional vector-space on which $Q$ acts on the right.

Definition 2.4.1. Define $\mathrm{GL}_{n} \times{ }^{Q} E:=\left(\mathrm{GL}_{n} \times E\right) / \sim$ where $\sim$ is the equivalence relation $(g, e) \sim(g q, e q)$ for every $g \in \mathrm{GL}_{n}, q \in Q$ and $e \in E$. Then $\pi_{E}$ : $\mathrm{GL}_{n} \times{ }^{Q} E \longrightarrow \mathrm{GL}_{n} / Q,(g, e) \mapsto g Q$, is a vector bundle called the vector bundle associated to $E$ (and the principal $Q$-bundle $\mathrm{GL}_{n} \longrightarrow \mathrm{GL}_{n} / Q$ ). For $g \in \mathrm{GL}_{n}, e \in E$, we write $[g, e] \in \mathrm{GL}_{n} \times{ }^{Q} E$ for the equivalence class of $(g, e) \in \mathrm{GL}_{n} \times E$ under $\sim$. We say that a vector bundle $\pi: \mathbf{E} \longrightarrow \mathrm{GL}_{n} / Q$ is homogeneous if $\mathbf{E}$ has a $\mathrm{GL}_{n}$-action and $\pi$ is $\mathrm{GL}_{n}$-equivariant, i.e, for every $y \in \mathbf{E}, \pi(g \cdot y)=g \cdot \pi(y)$.

Remark 2.4.2. There is a similar construction in the case when $E$ is a left $Q$ module.

In this section, we abbreviate $\mathrm{GL}_{n} \times{ }^{Q} E$ as $\widetilde{E}$. It is known that $\mathbf{E}$ is homogeneous if and only if $\mathbf{E} \simeq \widetilde{E}$ for some $Q$-module $E$. (If this is the case, then $E$ is the fiber of $\mathbf{E}$ over the coset $Q$.) A homogeneous bundle $\widetilde{E}$ is said to be irreducible (respectively indecomposable, completely reducible) if $E$ is an irreducible (respectively indecomposable, completely reducible) $Q$-module. It is known that $E$ is completely reducible if and only if $U_{Q}$ acts trivially and that $E$ is irreducible if and only if additionally it is irreducible as a representation of $L_{Q}$. See [Snow 1994, Section 5] or [Ottaviani 1995, Section 10] for the details.

Discussion 2.4.3. For the cohomology group computations in this paper, we will primarily be interested in the case when $\mathrm{GL}_{n} / Q$ is a Grassmannian. Thus let $Q=$ $P_{\hat{m}}$, with $1 \leq m \leq n-1$. A weight $\lambda$ is said to be $Q$-dominant if and only if when we express $\lambda$ as $\sum_{i=1}^{n} \lambda_{i} \epsilon_{i}$ (where $\epsilon_{i}$, for $1 \leq i \leq n$, is the character that sends a diagonal matrix in $T$ to its $i$-th entry), then $\lambda_{1} \geq \ldots \geq \lambda_{m}$ and $\lambda_{m+1} \geq \ldots \geq \lambda_{n}$. We will write $\lambda=\left(\lambda_{1}, \ldots, \lambda_{n}\right)$ to mean that $\lambda=\sum_{i=1}^{n} \lambda_{i} \epsilon_{i}$. Every finite-dimensional irreducible $Q$-module is of the form $H^{0}\left(Q / B_{n}, L_{\lambda}\right)$ for a $Q$-dominant weight $\lambda$. Hence the irreducible homogeneous vector bundles on $\mathrm{GL}_{n} / Q$ are in correspondence with $Q$-dominant weights. We describe them now. If $Q=P_{\widehat{n-i}}$, then $\mathrm{GL}_{n} / Q=\mathrm{Grass}_{i, n}$. (Recall that, for us, the $\mathrm{GL}_{n}$-action on $\mathbb{C}^{n}$ is on the right.) On Grass ${ }_{i, n}$, we have the tautological sequence

$$
0 \longrightarrow \mathcal{R}_{i} \longrightarrow \mathbb{C}^{n} \otimes \mathcal{O}_{\mathrm{Grass}_{i, n}} \longrightarrow \mathcal{Q}_{n-i} \longrightarrow 0
$$


of homogeneous vector bundles. The bundle $\mathcal{R}_{i}$ is called the tautological subbundle (of the trivial bundle $\mathbb{C}^{n}$ ) and $\mathcal{Q}_{n-i}$ is called the tautological quotient bundle. Every irreducible homogeneous bundle on Grass $i_{i, n}$ is of the form $S_{\left(\lambda_{1}, \cdots, \lambda_{n-i}\right)} \mathcal{Q}_{n-i}^{*} \otimes$ $S_{\left(\lambda_{n-i+1}, \cdots, \lambda_{n}\right)} \mathcal{R}_{i}^{*}$ for some $P_{\widehat{n-i}}$-dominant weight $\lambda$. Here $S_{\mu}$ denotes the Schur functor associated to the partition $\mu$ (cf. [Fulton and Harris 1991, §6.1]).

A $Q$-dominant weight is called $(m)$-dominant in [Weyman 2003, p. 114]. Although our definition looks like Weyman's definition, we should keep in mind that our action is on the right. We only have to be careful when we apply the Borel-Weil-Bott theorem (more specifically, the Bott algorithm). In this paper, our computations are done only on Grassmannians. If $\mu$ and $v$ are partitions, then $(\mu, v)$ will be $Q$-dominant (for a suitable $Q$ ), and will give us the vector bundle $S_{\mu} \mathcal{Q}^{*} \otimes S_{\nu} \mathcal{R}^{*}$ (this is where the right-action of $Q$ becomes relevant) and to compute its cohomology, we will have to apply the Bott algorithm to the $Q$ dominant weight $(\nu, \mu)$. (In [Weyman 2003], one would get $S_{\mu} \mathcal{R}^{*} \otimes S_{\nu} \mathcal{Q}^{*}$ and would apply the Bott algorithm to $(\mu, v)$.)

We now give a brief description of the Bott algorithm for computing the cohomology of irreducible homogeneous vector bundles on $\mathrm{GL}_{n} / Q$ [Weyman 2003, Remark 4.1.5].

Let $\alpha=\left(\alpha_{1}, \ldots, \alpha_{n}\right)$ be a weight. As in [Weyman 2003, Remark 4.1.5] we define an action of the permutation $v_{i}=(i, i+1)$ on the set of weights in the following way:

$$
v_{i} \alpha=\left(\alpha_{1}, \ldots, \alpha_{i-1}, \alpha_{i+1}-1, \alpha_{i}+1, \alpha_{i+2}, \ldots, \alpha_{n}\right) .
$$

The Bott algorithm may be applied to our case as follows. Let $Q=P_{\hat{m}}$, with $1 \leq m \leq n-1$ and let $\lambda=\left(\lambda_{1}, \ldots, \lambda_{n}\right)$ be a $Q$-dominant weight with associated homogeneous vector bundle $V(\lambda):=S_{\left(\lambda_{1}, \ldots, \lambda_{m}\right)} \mathcal{Q}^{*} \otimes S_{\left(\lambda_{m+1}, \ldots, \lambda_{n}\right)} \mathcal{R}^{*}$. We will apply the Bott algorithm to $\lambda^{\prime}=\left(\lambda_{m+1}, \ldots, \lambda_{n}, \lambda_{1}, \ldots, \lambda_{m}\right)$ in keeping with the last paragraph of Discussion 2.4.3.

If $\lambda^{\prime}$ is nonincreasing, then $H^{0}\left(\mathrm{GL}_{n} / Q, V(\lambda)\right)=S_{\lambda^{\prime}} \mathbb{C}^{n}$ and $H^{i}\left(\mathrm{GL}_{n} / Q, V(\lambda)\right)=0$ for $i>0$. Otherwise we start to apply the exchanges of type (2.4.5) to $\lambda^{\prime}$, trying to move smaller numbers on the left to the right. Two possibilities can occur:

(1) We apply an exchange of type (2.4.5) and it leaves the sequence unchanged. In this case $H^{i}\left(\mathrm{GL}_{n} / Q, V(\lambda)\right)=0$ for $i \geq 0$.

(2) After applying $j$ exchanges, we transform $\lambda^{\prime}$ into a nonincreasing sequence $\beta$. Then we have $H^{i}\left(\mathrm{GL}_{n} / Q, V(\lambda)\right)=0$ for $i \neq j$ and $H^{j}\left(\mathrm{GL}_{n} / Q, V(\lambda)\right)=S_{\beta} \mathbb{C}^{n}$.

\section{Properties of Schubert desingularization in type $\mathrm{C}$}

Recall the following result about the tangent space of a Schubert variety, see [Billey and Lakshmibai 2000, Chapter 4] for details. 
Proposition 3.1. Let $Q$ be a parabolic subgroup of $\mathrm{SL}_{2 n}$. Let $\tau \in W^{Q}$. Then the dimension of the tangent space of $X_{Q}(\tau)$ at $e_{\mathrm{id}}$ is

$$
\#\left\{s_{\alpha} \mid \alpha \in R^{-} \backslash R_{Q}^{-} \quad \text { and } \quad \tau \geq s_{\alpha} \text { in } W / W_{Q}\right\} .
$$

In particular, $X_{Q}(\tau)$ is smooth if and only if

$$
\operatorname{dim} X_{Q}(\tau)=\#\left\{s_{\alpha} \mid \alpha \in R^{-} \backslash R_{Q}^{-} \quad \text { and } \quad \tau \geq s_{\alpha} \text { in } W / W_{Q}\right\} .
$$

Notation 3.2. For an integer $i$ with $1 \leq i \leq n$ we define $i^{\prime}=2 n+1-i$. Let $1 \leq k<r \leq n$. Then

$$
\mathcal{W}_{k, r}= \begin{cases}\left(k+1, \ldots, r, n^{\prime}, \ldots,(r+1)^{\prime}, k^{\prime}, \ldots, 1^{\prime}\right) \in W^{P}, & \text { if } r<n \\ \left(k+1, \ldots, r, k^{\prime}, \ldots, 1^{\prime}\right) \in W^{P}, & \text { if } r=n\end{cases}
$$

Let $1 \leq k<r \leq n$ be integers. Let $w \in \mathcal{W}_{k, r}$ with $\tilde{w}$ its minimal representative in $W^{\widetilde{P}}$. Proposition 3.3. The Schubert variety $X_{\widetilde{Q}}(\tilde{w})$ in $H / \widetilde{Q}$ is smooth.

Proof. Let $w_{\max } \in W_{H}\left(=S_{2 n}\right)$ be the maximal representative of $\tilde{w}$. Then

$$
w_{\max }= \begin{cases}\left([r, k+1]\left[1^{\prime}, k^{\prime}\right]\left[(r+1)^{\prime}, n^{\prime}\right][n,(r+1)][k, 1]\left[(k+1)^{\prime}, r^{\prime}\right]\right), & \text { if } r<n, \\ \left([r, k+1]\left[1^{\prime}, k^{\prime}\right][k, 1]\left[(k+1)^{\prime}, r^{\prime}\right]\right), & \text { if } r=n .\end{cases}
$$

To see this we need to show that $X_{P_{\hat{i}}}\left(w_{\max }\right)=X_{P_{\hat{i}}}(\tilde{w})$ for $i=r-k, n, 2 n-(r-k)$ and that $w_{\max }$ is the maximal element of $W_{H}$ with this property. But this follows from the fact that for $\tau=\left(c_{1}, \ldots, c_{2 n}\right) \in W_{H}$ and $1 \leq i \leq 2 n$ we have that $X_{P_{\hat{i}}}(\tau)=X_{P_{\hat{i}}}\left(\tau^{\prime}\right)$ where $\tau^{\prime} \in W^{P_{\hat{i}}}$ is the element with $c_{1}, \ldots, c_{i}$ written in increasing order.

Thus $X_{B_{H}}\left(w_{\max }\right)$ is the inverse image of $X_{\widetilde{Q}}(\tilde{w})$ under the natural morphism $H / B_{H} \rightarrow H / \widetilde{Q}$. As $w_{\max }$ is a 4231 and 3142 avoiding element of $W_{H}$ we have that $X_{B_{H}}\left(w_{\max }\right)$ is nonsingular (see [Billey and Lakshmibai 2000, 8.1.1]). Since the morphism $H / B_{H} \rightarrow H / \widetilde{Q}$ has nonsingular fibers (namely $\left.\widetilde{Q} / B_{H}\right), X_{\widetilde{Q}}(\tilde{w})$ must be smooth.

Proposition 3.4. The Schubert variety $X_{\widetilde{P}}(\tilde{w})$ in $G / \widetilde{P}$ is smooth .

Proof. Let $w_{\max }$ be as in the proof of Proposition 3.3. Then clearly $w_{\max }$ is in $W_{G}$ and $X_{B_{G}}\left(w_{\max }\right)$ is the inverse image of $X_{\widetilde{P}}(\tilde{w})$ under the natural morphism $G / B_{G} \rightarrow G / \widetilde{P}$.

Claim. $X_{B_{G}}\left(w_{\max }\right)$ is smooth.

Note that the claim implies the required result (since the canonical morphism $G / B_{G} \rightarrow G / \widetilde{P}$ is a fibration with nonsingular fibers (namely, $\left.\widetilde{P} / B_{G}\right)$ ). To prove the claim, as seen in the proof of Proposition 3.3, we have that $X_{B_{H}}\left(w_{\max }\right)$ is smooth. 
We conclude the smoothness of $X_{B_{G}}\left(w_{\max }\right)$ using the following two formulas [Lakshmibai 1987, §3(VI), Remark 5.8]:

$$
l_{G}(\theta)=\frac{1}{2}\left[l_{H}(\theta)+m(\theta)\right],
$$

where we let $\theta \in W_{G}$, say, $\theta=\left(a_{1}, \cdots a_{n}\right)$. With $m(\theta)=\#\left\{i, 1 \leq i \leq m \mid a_{i}>m\right\}$ (cf. (2.2.2)), we have

$$
\operatorname{dim} T_{\text {id }}(\theta, G)=\frac{1}{2}\left[\operatorname{dim} T_{\text {id }}(\theta, H)+c(\theta)\right],
$$

where $c(\theta)=\#\left\{1 \leq i \leq m \mid \theta \geq s_{\epsilon_{2 i}}\right\}$, and $T_{\mathrm{id}}(\theta, G)$ (resp $\left.T_{\mathrm{id}}(\theta, H)\right)$ denotes the Zariski tangent space of $X_{B_{G}}(\theta)\left(\operatorname{resp} X_{B_{H}}(\theta)\right)$ at $e_{\text {id }}$. Note that $s_{\epsilon_{2 i}}$ is just the transposition $\left(i, i^{\prime}\right)$ (cf. (2.2.1)). Now taking $\theta=w_{\max }$, we have, $c\left(w_{\max }\right)=m\left(w_{\max }\right)$. Hence we obtain from (1), (2) that $\operatorname{dim} T_{\mathrm{id}}\left(w_{\max }, G\right)=l_{G}\left(w_{\max }\right)$, proving that $X_{B_{G}}\left(w_{\max }\right)$ is smooth at $e_{\mathrm{id}}$, and hence is nonsingular (note that for a Schubert variety $X$, the singular locus of $X, \operatorname{Sing}(X)$, is $B$-stable implying $e_{\mathrm{id}} \in \operatorname{Sing}(X)$ if $\operatorname{Sing}(X) \neq \varnothing)$. Thus the claim (and hence the required result) follows.

Remark 3.5. We have that $X_{\widetilde{P}}(\tilde{w})$ is the fixed point set under an automorphism of order two of the Schubert variety $X_{\widetilde{Q}}(\tilde{w})$ and thus is smooth, provided char $K \neq 2$ ([Edixhoven 1992, Proposition 3.4]).

Discussion 3.6. To give a characterization of $Y_{\widetilde{Q}}(\tilde{w})$ we first need a review of the structure of $O_{H / \widetilde{Q}}^{-}$and its Plücker coordinates.

Recall that for the Plücker embedding of the Grassmannian Grass $d_{d, n}$, the Plücker coordinate $p_{\underline{i}}(U), U \in \operatorname{Grass}_{d, n}$ and $\underline{i}=\left(i_{1}, \ldots, i_{d}\right)$ with $1 \leq i_{1}<\ldots<i_{d}<n$, is just the $d \times d$ minor of the matrix $A_{n \times d}$ with row indices $\left(i_{1}, \ldots, i_{d}\right)$ (here the matrix $A_{n \times d}$ represents the $d$-dimensional subspace $U$ with respect to the standard basis).

The cell $O_{H / \widetilde{Q}}^{-}$can be identified with the affine space of lower-triangular matrices with possible nonzero entries $x_{i j}$ at row $i$ and column $j$ where $(i, j)$ is such that there exists an $l \in\{r-k, n, 2 n-(r-k)\}$ such that $j \leq l<i \leq N$. To see this, note that we are interested in those $(i, j)$ such that the root $\epsilon_{i}-\epsilon_{j}$ belongs to $R^{-} \backslash R_{\widetilde{Q}}^{-}$. Since $R_{\widetilde{Q}}^{-}=R_{Q_{r-k}}^{-} \cap R_{Q_{\hat{n}}} \cap R_{Q_{2 n-(r-k)}}$, we see that we are looking for $(i, j)$ such that $\epsilon_{i}-\epsilon_{j} \in R^{-} \backslash R_{Q_{\hat{\imath}}}^{-}$, for some $l \in\{r-k, n, 2 n-(r-k)\}$. For the maximal parabolic subgroup $P_{\hat{l}}$, we have, $R^{-} \backslash R_{Q_{\hat{l}}}^{-}=\left\{\epsilon_{i}-\epsilon_{j} \mid 1 \leq j \leq l<i \leq N\right\}$. We have $\operatorname{dim} O_{H / \widetilde{Q}}^{-}=\left|R^{-} \backslash R_{\widetilde{Q}}^{-}\right|$.

Thus we have the following identification

$$
O_{H / \widetilde{Q}}^{-}=\left[\begin{array}{cccc}
\operatorname{Id}_{r-k} & 0 & 0 & 0 \\
A^{\prime} & \operatorname{Id}_{n-(r-k)} & 0 & 0 \\
\mathcal{D}_{1} & \mathcal{D}_{2} & \operatorname{Id}_{n-(r-k)} & 0 \\
\mathcal{D}_{3} & \mathcal{D}_{4} & E^{\prime} & \operatorname{Id}_{r-k}
\end{array}\right]
$$


where the block matrices have possible nonzero entries $x_{i j}$ given by

$$
\begin{array}{rlrl}
A^{\prime} & =\left[\begin{array}{ccc}
x_{(r-k)+1} & \ldots & x_{(r-k)+1} r-k \\
\vdots & & \vdots \\
x_{n} 1 & \ldots & x_{n r-k}
\end{array}\right], & E^{\prime}=\left[\begin{array}{ccc}
x_{2 n-(r-k)+1} n+1 & \ldots & x_{2 n-(r-k)+1} 2 n-(r-k) \\
\vdots & & \vdots \\
x_{2 n} n+1 & \ldots & x_{2 n 2 n-(r-k)}
\end{array}\right], \\
\mathcal{D}_{1}=\left[\begin{array}{ccc}
x_{n+1} 1 & \ldots & x_{n+1} r-k \\
\vdots & & \vdots \\
x_{2 n-(r-k)} & \ldots & x_{2 n-(r-k) r-k}
\end{array}\right], & \mathcal{D}_{2}=\left[\begin{array}{ccc}
x_{n+1}(r-k)+1 & \ldots & x_{n+1} n \\
\vdots & & \vdots \\
x_{2 n-(r-k)(r-k)+1} & \ldots & x_{2 n-(r-k) n}
\end{array}\right], \\
\mathcal{D}_{3}=\left[\begin{array}{ccc}
x_{2 n-(r-k)+1} & \ldots & x_{2 n-(r-k)+1} r-k \\
\vdots & & \vdots \\
x_{2 n} 1 & \ldots & x_{2 n r-k}
\end{array}\right], & \mathcal{D}_{4}=\left[\begin{array}{ccc}
x_{2 n-(r-k)+1(r-k)+1} & \ldots & x_{2 n-(r-k)+1 n} \\
\vdots & & \vdots \\
x_{2 n(r-k)+1} & \ldots & x_{2 n n}
\end{array}\right] .
\end{array}
$$

We may break the Plücker coordinates we want to understand into several cases. Case 1: For $i>r, j \leq r-k$ the Plücker coordinate $p_{(i, j)}^{(r-k)}$ on the Grassmannian $H / Q_{\widehat{r-k}}$ lifts to a regular function on $H / \widetilde{Q}$. Its restriction to $O_{H / \widetilde{Q}}^{-}$is the $r-$ $k \times r-k$ minor of (3.7) with column indices $\{1,2, \ldots, r-k\}$ and row indices $\{1, \ldots, j-1, j+1, \ldots, r-k, i\}$. This minor is the determinant of an $r-k \times r-k$ matrix with the top $(r-k)-1$ rows equal to $\operatorname{Id}_{r-k}$ omitting the $j$-th row, and the bottom row equal to the first $r-k$ entries of the $i$-th row of (3.7). The determinant of this matrix is thus $(-1)^{(r-k)-j} x_{i j}$. Thus for $i>r, j \leq r-k$ :

$$
\left.p_{(i, j)}^{(r-k)}\right|_{O_{H / \tilde{Q}}^{-}}=(-1)^{(r-k)-j} x_{i j} .
$$

Case 2: For $i>2 n-(r-k), n<j \leq 2 n-(r-k)$ the Plücker coordinate $p_{(i, j)}^{(2 n-(r-k))}$ on the Grassmannian $H / Q_{2 n-(r-k)}$ lifts to a regular function on $H / \widetilde{Q}$. Its restriction to $O_{H / \widetilde{Q}}^{-}$is the $2 n-(r-k) \times 2 n-(r-k)$ minor of (3.7) with column indices $\{1,2, \ldots, 2 n-(r-k)\}$ and row indices $\{1, \ldots, j-1, j+1, \ldots, 2 n-(r-k), i\}$. This minor is the determinant of

$$
\left[\begin{array}{ccc}
\operatorname{Id}_{r-k} & 0 & 0 \\
A^{\prime} & \operatorname{Id}_{n-(r-k)} & 0 \\
\widehat{\mathcal{D}}_{1} & \widehat{\mathcal{D}}_{2} & \widehat{I}_{1} \\
{\left[x_{i 1} \ldots x_{i r-k}\right]} & {\left[x_{i(r-k)+1} \ldots x_{i n}\right]} & {\left[x_{i n+1} \ldots x_{i 2 n-(r-k)}\right]}
\end{array}\right]
$$

where $\widehat{\mathcal{D}}_{1}, \widehat{\mathcal{D}}_{2}$, and $\widehat{I}_{1}$ are equal to, respectively, $\mathcal{D}_{1}, \mathcal{D}_{2}$, and $\operatorname{Id}_{n-(r-k)}$ with their $(j-n)$-th rows omitted. The determinant of (3.9) is equal to the determinant of

$$
\left[\begin{array}{c}
\widehat{I_{1}} \\
{\left[x_{i n+1} \ldots x_{i 2 n-(r-k)}\right]}
\end{array}\right] \text {. }
$$

As above this is just an identity matrix with a single row replaced and so its determinant is just $(-1)^{2 n-(r-k)-j} x_{i j}$. Thus for $i>2 n-(r-k), n<j \leq 2 n-(r-k)$ :

$$
\left.p_{(i, j)}^{(2 n-(r-k))}\right|_{O_{H / \tilde{Q}}^{-}}=(-1)^{2 n-(r-k)-j} x_{i j} .
$$


Case 3: For $i>2 n-(r-k), r-k<j \leq n$ the Plücker coordinate $p_{(i, j)}^{(2 n-(r-k))}$ on the Grassmannian $H / Q_{2 n-(r-k)}$ lifts to a regular function on $H / \widetilde{Q}$. Its restriction to $O_{H / \widetilde{Q}}^{-}$is the $2 n-(r-k) \times 2 n-(r-k)$ minor of (3.7) with column indices $\{1,2, \ldots, 2 n-(r-k)\}$ and row indices $\{1, \ldots, j-1, j+1, \ldots, 2 n-(r-k), i\}$. This minor is the determinant of

$$
\left[\begin{array}{ccc}
\mathrm{Id}_{r-k} & 0 & 0 \\
\widehat{A}^{\prime} & \widehat{I}_{2} & 0 \\
\mathcal{D}_{1} & \mathcal{D}_{2} & \operatorname{Id}_{n-(r-k)} \\
{\left[x_{i} 1 \ldots x_{i r-k}\right]} & {\left[x_{i}(r-k)+1 \ldots x_{i n}\right]} & {\left[x_{i n+1} \ldots x_{i 2 n-(r-k)}\right]}
\end{array}\right]
$$

where $\widehat{A^{\prime}}$ and $\widehat{I_{2}}$ are equal to, respectively, $A^{\prime}$ and $\operatorname{Id}_{n-(r-k)}$ with their $j-(r-k)$-th rows omitted. The determinant of (3.11) is equal to the determinant of

$$
\left[\begin{array}{cc}
\widehat{I_{2}} & 0 \\
\mathcal{D}_{2} & \operatorname{Id}_{n-(r-k)} \\
{\left[x_{i(r-k)+1} \ldots x_{i n}\right]} & {\left[x_{i n+1} \ldots x_{i 2 n-(r-k)}\right]}
\end{array}\right] .
$$

To calculate this, shift the bottom row so that it becomes the $j-(r-k)$-th row of $\widehat{I_{2}}$. Let $M=2 n-(r-k)-j$. Then the determinant of (3.12) will be $(-1)^{M}$ times the determinant of

$$
\left[\begin{array}{cc}
I_{3} & Z \\
\mathcal{D}_{2} & \operatorname{Id}_{n-(r-k)}
\end{array}\right]
$$

where $I_{3}$ is $\operatorname{Id}_{n-(r-k)}$ with the $j-(r-k)$-th row replaced by $\left[x_{i(r-k)+1} \ldots x_{i n}\right]$ and $Z$ is the zero matrix with the $j-(r-k)$-th row replaced by $\left[x_{i n+1} \ldots x_{i 2 n-(r-k)}\right]$. Since the lower right block matrix of (3.13) commutes with its lower left block matrix we have that the determinant of (3.13) is equal to the determinant of $I_{3}-Z \mathcal{D}_{2}$. We have that $Z \mathcal{D}_{2}$ is equal to the zero matrix with its $j-(r-k)$-th row replaced by

$$
\left[x_{i(r-k)+1} \ldots x_{i n}\right] \mathcal{D}_{2} \text {. }
$$

And thus $I_{3}-Z \mathcal{D}_{2}$ is equal to $\operatorname{Id}_{n-(r-k)}$ with the $j-(r-k)$-th row replaced by

$$
\left[x_{i(r-k)+1} \ldots x_{i n}\right]-\left[x_{i(r-k)+1} \ldots x_{i n}\right] \mathcal{D}_{2} \text {. }
$$

And so the determinant of $I_{3}-Z \mathcal{D}_{2}$ is merely equal to the $j-(r-k)$-th entry of $I_{3}-Z \mathcal{D}_{2}$ which is

$$
x_{i j}-\left[x_{i(r-k)+1} \ldots x_{i n}\right]\left[x_{n+1 j} \ldots x_{2 n-(r-k) j}\right]^{T} .
$$

Combining all our steps, we finally have that for $i>2 n-(r-k), r-k<j \leq n$ : (3.14) $\left.p_{(i, j)}^{(2 n-(r-k))}\right|_{O_{H / \widetilde{Q}}^{-}}=(-1)^{M}\left(x_{i j}-\left[x_{i(r-k)+1} \ldots x_{i n}\right]\left[x_{n+1 j} \ldots x_{2 n-(r-k) j}\right]^{T}\right)$. 
Theorem 3.15. The opposite cell $Y_{\widetilde{Q}}(\tilde{w})$ can be identified with the subspace of $\mathrm{O}_{\mathrm{H} / \widetilde{Q}}^{-}$given by matrices of the form

$$
\left[\begin{array}{cccc}
\mathrm{Id}_{r-k} & 0 & 0 & 0 \\
A^{\prime} & \mathrm{Id}_{n-(r-k)} & 0 & 0 \\
0 & \mathcal{D}_{2} & \operatorname{Id}_{n-(r-k)} & 0 \\
0 & E^{\prime} \mathcal{D}_{2} & E^{\prime} & \operatorname{Id}_{r-k}
\end{array}\right]
$$

with $\mathcal{D}_{2} \in \operatorname{Mat}_{n-(r-k)}, A^{\prime} \in \operatorname{Mat}_{n-(r-k) \times r-k}$ with the bottom $n-r$ rows of $A^{\prime}$ all $z e r o$, and $E^{\prime} \in \mathrm{Mat}_{r-k \times n-(r-k)}$ with the left $n-r$ columns of $E^{\prime}$ all zero.

Proof. For $j \leq r-k<i$ the reflection $(i, j)$ equals $(1,2, \ldots, j-1, j+1, \ldots, r-$ $k, i)$ and $\tilde{w}$ equals $(k+1, \ldots, r)$ in $W / W_{Q_{r-k}}$. Thus for $i>r$ and $j \leq r-k$, the reflection $(i, j)$ is not smaller than $\tilde{w}$ in $W / W_{Q_{r-k}}$ so the Plücker coordinate $p_{(i, j)}^{(r-k)}$ vanishes on $X_{\widetilde{Q}}(\tilde{w})$. We saw in (3.8) that for such $(i, j)$ we have $p_{(i, j)}^{(r-k)}=$ $(-1)^{(r-k)-j} x_{i j}$ and thus $x_{i j} \equiv 0$ on $Y_{\widetilde{Q}}(\tilde{w})$.

For $j \leq n<i$ the reflection $(i, j)$ equals $(1,2, \ldots, j-1, j+1, \ldots, n, i)$ and $\tilde{w}$ is equal to $\left(k+1, \ldots, r, n^{\prime}, \ldots,(r+1)^{\prime}, k^{\prime}, \ldots, 1^{\prime}\right)$ in $W / W_{Q_{\hat{n}}}$. Thus there is no choice of $(i, j)$ such that $(i, j)$ is not smaller than $\tilde{w}$ in $W / W_{Q_{\hat{n}}}$.

For $j \leq 2 n-(r-k)<i$ the reflection $(i, j)$ equals $(1,2, \ldots, j-1, j+1, \ldots, 2 n-$ $(r-k), i)$ and $\tilde{w}$ equals $\left(1, \ldots, n, n^{\prime}, \ldots,(r+1)^{\prime}, k^{\prime}, \ldots, 1^{\prime}\right)$ in $W / W_{Q_{2 n-(r-k)}}$. Thus for $i>2 n-(r-k)$, and $j \leq 2 n-r$ the reflection $(i, j)$ is not smaller than $\tilde{w}$ in $W / W_{Q_{2 n-(r-k)}}$. We break these into two cases, ignoring those $j \leq r-k$ as we have already shown above that for $j \leq r-k$ and $i>2 n-(r-k)$ we have $x_{i j} \equiv 0$ on $Y_{\widetilde{Q}}(\tilde{w})$.

The first case is for $(i, j)$ with $i>2 n-(r-k)$, and $n<j \leq 2 n-r$. The fact that $(i, j)$ is not smaller than $\tilde{w}$ in $W / W_{Q_{2 n-(r-k)}}$ implies that the Plücker coordinate $p_{(i, j)}^{(2 n-(r-k))}$ vanishes on $X_{\widetilde{Q}}(\tilde{w})$. We saw in (3.10) that for such $(i, j)$ we have $p_{(i, j)}^{(2 n-(r-k))}=(-1)^{2 n-(r-k)-j} x_{i j}$ and thus $x_{i j} \equiv 0$ on $Y_{\widetilde{Q}}(\tilde{w})$.

The second case is for $(i, j)$ with $i>2 n-(r-k)$ and $r-k<j \leq n$. The reflection $(i, j)$ is not smaller than $\tilde{w}$ in $W / W_{Q_{2 n-(r-k)}}$ implies that the Plücker coordinate $p_{(i, j)}^{(2 n-(r-k))}$ vanishes on $X_{\widetilde{Q}}(\tilde{w})$. We saw in (3.14) that for such $(i, j)$ we have $p_{(i, j)}^{(2 n-(r-k))}=(-1)^{M}\left(x_{i j}-\left[x_{i(r-k)+1} \ldots x_{i n}\right]\left[x_{n+1 j} \ldots x_{2 n-(r-k) j}\right]^{T}\right)$. Combining these two facts we get $x_{i j}=\left[x_{i(r-k)+1} \ldots x_{i n}\right]\left[x_{n+1} \ldots x_{2 n-(r-k) j}\right]^{T}$.

As $\left[x_{i(r-k)+1} \ldots x_{i n}\right]$ is the $(2 n-(r-k)-i)$-th row of $E^{\prime}$ and $\left[x_{n+1} \ldots x_{2 n-(r-k) j}\right]^{T}$ is the $(2 n-(r-k)-j)$-th column of $\mathcal{D}_{2}$ it is clear that on $Y_{\widetilde{Q}}(\tilde{w})$ we have $x_{i j}=\left(E^{\prime} X\right)_{(2 n-(r-k)-i)(2 n-(r-k)-j)}$.

On the other hand note that the reflections $(i, j)$ with $i>r$ and $j \leq r-k$, and $i>2 n-(r-k)$ and $r-k<j \leq 2 n-r$ are precisely the reflections $s_{\alpha}$ with $\alpha \in R^{-} \backslash R_{\widetilde{Q}}^{-}$and $\tilde{w} \ngtr s_{\alpha}$ in $W / W_{\widetilde{Q}}$. Since $X_{\widetilde{Q}}(\tilde{w})$ is smooth this implies by Proposition 3.1 that the codimension of $Y_{\widetilde{Q}}(\tilde{w})$ in $O_{H / \widetilde{Q}}^{-}$is equal to 
$\#\{(i, j) \mid i>r$ and $j \leq r-k$, or $i>2 n-(r-k)$ and $r-k<j \leq 2 n-r\}$. Above we have shown that for each such $(i, j), x_{i j}$ either vanishes, or is completely dependent on the entries of $E^{\prime} X$. Thus $Y_{\widetilde{Q}}(\tilde{w})$ is the subspace of $O_{H / \widetilde{Q}}^{-}$defined by the vanishing of $\left\{x_{i j} \mid i>r\right.$ and $j \leq r-k$, or $i>2 n-(r-k)$ and $\left.n<j \leq 2 n-r\right\}$ and $x_{i j}=\left(E^{\prime} X\right)_{(2 n-(r-k)-i)(2 n-(r-k)-j)}$ for $i>2 n-(r-k)$ and $r-k<j \leq n$.

Example 3.16. Let $k=2, r=4$, and $n=5$. Then $\widetilde{Q}=Q_{\hat{2}, \hat{5}, \hat{8}}, w=(3,4,6,9,10)$, and $\tilde{w}=(3,4,6,9,10,1,2,5)$. Then

$$
O_{H / \widetilde{Q}}^{-}=\left[\begin{array}{cccccccccc}
1 & 0 & 0 & 0 & 0 & 0 & 0 & 0 & 0 & 0 \\
0 & 1 & 0 & 0 & 0 & 0 & 0 & 0 & 0 & 0 \\
x_{31} & x_{32} & 1 & 0 & 0 & 0 & 0 & 0 & 0 & 0 \\
x_{41} & x_{42} & 0 & 1 & 0 & 0 & 0 & 0 & 0 & 0 \\
x_{51} & x_{52} & 0 & 0 & 1 & 0 & 0 & 0 & 0 & 0 \\
x_{61} & x_{62} & x_{63} & x_{64} & x_{65} & 1 & 0 & 0 & 0 & 0 \\
x_{71} & x_{72} & x_{73} & x_{74} & x_{75} & 0 & 1 & 0 & 0 & 0 \\
x_{81} & x_{82} & x_{83} & x_{84} & x_{85} & 0 & 0 & 1 & 0 & 0 \\
x_{91} & x_{92} & x_{93} & x_{94} & x_{95} & x_{96} & x_{97} & x_{98} & 1 & 0 \\
x_{101} & x_{102} & x_{103} & x_{104} & x_{105} & x_{106} & x_{107} & x_{108} & 0 & 1
\end{array}\right] .
$$

And $Y_{\widetilde{P}}(\tilde{w})$ will be the subspace of $O_{H / \widetilde{Q}}^{-}$given by

$\left[\begin{array}{cccccccccc}1 & 0 & 0 & 0 & 0 & 0 & 0 & 0 & 0 & 0 \\ 0 & 1 & 0 & 0 & 0 & 0 & 0 & 0 & 0 & 0 \\ x_{31} & x_{32} & 1 & 0 & 0 & 0 & 0 & 0 & 0 & 0 \\ x_{41} & x_{42} & 0 & 1 & 0 & 0 & 0 & 0 & 0 & 0 \\ 0 & 0 & 0 & 0 & 1 & 0 & 0 & 0 & 0 & 0 \\ 0 & 0 & x_{63} & x_{64} & x_{65} & 1 & 0 & 0 & 0 & 0 \\ 0 & 0 & x_{73} & x_{74} & x_{75} & 0 & 1 & 0 & 0 & 0 \\ 0 & 0 & x_{83} & x_{84} & x_{85} & 0 & 0 & 1 & 0 & 0 \\ 0 & 0 & x_{97} x_{73}+x_{98} x_{83} & x_{97} x_{74}+x_{98} x_{84} & x_{97} x_{75}+x_{98} x_{85} & 0 & x_{97} & x_{98} & 1 & 0 \\ 0 & 0 & x_{107} x_{73}+x_{108} x_{83} & x_{107} x_{74}+x_{108} x_{84} & x_{107} x_{75}+x_{108} & 0 & x_{107} & x_{108} & 0 & 1\end{array}\right]$.

Corollary 3.17. The opposite cell $Y_{\widetilde{P}}(\tilde{w})$ can be identified with the subspace of $O_{G / \widetilde{P}}^{-}$given by matrices of the form

$$
\left[\begin{array}{cccc}
\mathrm{Id}_{r-k} & 0 & 0 & 0 \\
A^{\prime} & \mathrm{Id}_{n-(r-k)} & 0 & 0 \\
0 & \mathcal{D}_{2} & \operatorname{Id}_{n-(r-k)} & 0 \\
0 & -J\left(A^{\prime}\right)^{T} J \mathcal{D}_{2} & -J\left(A^{\prime}\right)^{T} J & \mathrm{Id}_{r-k}
\end{array}\right]
$$

with $J \mathcal{D}_{2} \in \operatorname{Sym}_{n-(r-k)}$ and $A^{\prime} \in \operatorname{Mat}_{n-(r-k) \times r-k}$ with the bottom $n-r$ rows of $A^{\prime}$ all zero. 
Proof. Let $y \in Y_{\widetilde{P}}(\tilde{w})=\left(Y_{\widetilde{Q}}(\tilde{w})\right)^{\sigma} \subset Y_{\widetilde{Q}}(\tilde{w})$. So $y$ is just an element of $Y_{\widetilde{Q}}(\tilde{w})$ that is fixed under the involution $\sigma$. That is, an element which satisfies (2.3.1)-(2.3.3). Theorem 3.15 gives us that $y$ is of the form

$$
\left[\begin{array}{cccc}
\mathrm{Id}_{r-k} & 0 & 0 & 0 \\
A^{\prime} & \mathrm{Id}_{n-(r-k)} & 0 & 0 \\
0 & \mathcal{D}_{2} & \mathrm{Id}_{n-(r-k)} & 0 \\
0 & E^{\prime} \mathcal{D}_{2} & E^{\prime} & \mathrm{Id}_{r-k}
\end{array}\right]
$$

with $\mathcal{D}_{2} \in$ Mat $_{n-(r-k)}, A^{\prime} \in$ Mat $_{n-(r-k) \times r-k}$ with the bottom $n-r$ rows of $A^{\prime}$ all zero, and $E^{\prime} \in \mathrm{Mat}_{r-k \times n-(r-k)}$ with the left $n-r$ columns of $E^{\prime}$ all zero. We must now check what restrictions on $y$ are required for it to satisfy (2.3.1)-(2.3.3). For $y$ to satisfy (2.3.3) we know that

$$
\left[\begin{array}{cc}
\mathrm{Id}_{r-k} & 0 \\
A^{\prime} & \mathrm{Id}_{n-(r-k)}
\end{array}\right]^{T}\left[\begin{array}{ll}
0 & J \\
J & 0
\end{array}\right]\left[\begin{array}{cc}
\mathrm{Id}_{r-k} & 0 \\
E^{\prime} & \operatorname{Id}_{n-(r-k)}
\end{array}\right]\left(=\left[\begin{array}{cc}
\left(A^{\prime}\right)^{T} J+J E^{\prime} & J \\
J & 0
\end{array}\right]\right)
$$

must equal

$$
\left[\begin{array}{ll}
0 & J \\
J & 0
\end{array}\right]
$$

which implies that $E^{\prime}=-J\left(A^{\prime}\right)^{T} J$.

Any $y$ clearly satisfies (2.3.2). And finally for $y$ to satisfy (2.3.1),

$$
\left[\begin{array}{cc}
0 & \mathcal{D}_{2} \\
0 & -J\left(A^{\prime}\right)^{T} J \mathcal{D}_{2}
\end{array}\right]^{T}\left[\begin{array}{ll}
0 & J \\
J & 0
\end{array}\right]\left[\begin{array}{cc}
\operatorname{Id}_{r-k} & 0 \\
A^{\prime} & \operatorname{Id}_{n-(r-k)}
\end{array}\right]\left(=\left[\begin{array}{cc}
0 & 0 \\
0 & \mathcal{D}_{2}^{T} J
\end{array}\right]\right)
$$

must equal

$$
\left[\begin{array}{cc}
\operatorname{Id}_{r-k} & 0 \\
A^{\prime} & \operatorname{Id}_{n-(r-k)}
\end{array}\right]^{T}\left[\begin{array}{ll}
0 & J \\
J & 0
\end{array}\right]\left[\begin{array}{cc}
0 & \mathcal{D}_{2} \\
0 & -J\left(A^{\prime}\right)^{T} J \mathcal{D}_{2}
\end{array}\right]\left(=\left[\begin{array}{cc}
0 & 0 \\
0 & J \mathcal{D}_{2}
\end{array}\right]\right)
$$

which implies that $J \mathcal{D}_{2}=\mathcal{D}_{2}^{T} J$, or equivalently $J \mathcal{D}_{2} \in \operatorname{Sym}_{n-(r-k)}$.

Remark 3.18. We may identify $O_{P / \widetilde{P}}^{-}$with $O_{\mathrm{GL}_{n} / P_{r-k}^{\prime}}^{-}$under the map

$$
\left[\begin{array}{cc}
\mathcal{A} & 0 \\
0 & J\left(\mathcal{A}^{T}\right)^{-1} J
\end{array}\right] \mapsto \mathcal{A}
$$

Remark 3.19. Let $V_{w}$ be the linear subspace of $\operatorname{Sym}_{n}$ given by $x_{i j}=0$ if $j \leq r-k$ or $i<n-(r-k)$. And let $V_{w}^{\prime}$ be the linear subspace of $O_{\mathrm{GL}_{n} / P_{r-k}^{\prime}}^{-}$given by $x_{i j}=0$ if $i>r$ and $j \leq r-k$.

Consider the map $\delta: Y_{\widetilde{P}}(\tilde{w}) \hookrightarrow O_{G / \widetilde{P}}^{-}=O_{G / P}^{-} \times O_{P / \widetilde{P}}^{-} \cong O_{G / P}^{-} \times O_{\mathrm{GL}_{n} / P^{\prime}}^{-}$, where the first map is inclusion, the second is simply the product decomposition, 
and the final map is from Remark 3.18. This map is given explicitly by

$$
\begin{gathered}
{\left[\begin{array}{cccc}
\mathrm{Id}_{r-k} & 0 & 0 & 0 \\
A^{\prime} & \operatorname{Id}_{n-(r-k)} & 0 & 0 \\
0 & \mathcal{D}_{2} & \mathrm{Id}_{n-(r-k)} & 0 \\
0 & -J\left(A^{\prime}\right)^{T} J \mathcal{D}_{2} & -J\left(A^{\prime}\right)^{T} J & \mathrm{Id}_{r-k}
\end{array}\right] \mapsto} \\
\left(\left[\begin{array}{cccc}
\mathrm{Id}_{r-k} & 0 & 0 & 0 \\
0 & \mathrm{Id}_{n-(r-k)} & 0 & 0 \\
-\mathcal{D}_{2} A^{\prime} & \mathcal{D}_{2} & \mathrm{Id}_{n-(r-k)} & 0 \\
J\left(A^{\prime}\right)^{T} J \mathcal{D}_{2} A^{\prime} & -J\left(A^{\prime}\right)^{T} J \mathcal{D}_{2} & 0 & \mathrm{Id}_{r-k}
\end{array}\right],\left[\begin{array}{cc}
\operatorname{Id}_{r-k} & 0 \\
A^{\prime} & \mathrm{Id}_{n-(r-k)}
\end{array}\right]\right)
\end{gathered}
$$

Consider the isomorphism $\gamma: O_{G / P}^{-} \times O_{\mathrm{GL}_{n} / P_{r-k}^{\prime}}^{-} \rightarrow \operatorname{Sym}_{n} \times O_{\mathrm{GL}_{n} / P_{r-k}^{\prime}}^{-}(\mathrm{cf}$. Remark 2.2.6) given by

$$
\left(\left[\begin{array}{cc}
\operatorname{Id}_{n} & 0 \\
L & \operatorname{Id}_{n}
\end{array}\right],\left[\begin{array}{cc}
\operatorname{Id}_{r-k} & 0 \\
N & \operatorname{Id}_{n-(r-k)}
\end{array}\right]\right) \mapsto\left((L N)^{T} J N,\left[\begin{array}{cc}
\operatorname{Id}_{r-k} & 0 \\
N & \operatorname{Id}_{n-(r-k)}
\end{array}\right]\right) .
$$

We have that under the map $\gamma \circ \delta, Y_{\widetilde{P}}(\tilde{w})$ gets identified with $V_{w} \times V_{w}^{\prime}$. This follows by a simple computation and Corollary 3.17.

Definition 3.20. Now let $Z_{\widetilde{P}}(\tilde{w}):=Y_{P}(w) \times_{X_{P}(w)} X_{\widetilde{P}}(\tilde{w})$. Then $Z_{\widetilde{P}}(\tilde{w})=\left(O_{G / P}^{-} \times\right.$ $P / \widetilde{P}) \cap X_{\widetilde{P}}(\tilde{w})$. Hence $Z_{\widetilde{P}}(\tilde{w})$ is smooth, being open in the smooth $X_{\widetilde{P}}(\tilde{w})$ (cf. Proposition 3.3).

Write $p$ for the composite map $Z_{\widetilde{P}}(\tilde{w}) \rightarrow O_{G / P}^{-} \times P / \widetilde{P} \rightarrow P / \widetilde{P}\left(\cong \mathrm{GL}_{n} / P_{r-k}^{\prime}\right)$ where the first map is the inclusion and the second map is the projection. Using Proposition 2.3.4(c) and (d) we see that

$$
p\left(\left[\begin{array}{cc}
A & 0 \\
D & J\left(A^{T}\right)^{-1} J
\end{array}\right](\bmod \widetilde{P})\right)=A\left(\bmod P_{r-k}^{\prime}\right) .
$$

Note that $A$ is invertible by $2.3 .4(\mathrm{~b})$.

Using the injective map

$$
A \in B_{n} \longmapsto\left[\begin{array}{cc}
A & 0_{n \times n} \\
0_{n \times n} & J\left(A^{T}\right)^{-1} J
\end{array}\right] \in B_{G},
$$

$B_{n}$ can be thought of as a subgroup of $B_{G}$. With this identification we have the following proposition.

Proposition 3.21. $Z_{\widetilde{P}}(\tilde{w})$ is $B_{n}$-stable for the action on the left by multiplication. Further $p$ is $B_{n}$ equivariant.

Proof. Let $z \in \mathrm{SP}_{2 n}$ such that $z \widetilde{P} \in Z_{\widetilde{P}}(\tilde{w})$. Then by Proposition 2.3.4(c) we may write

$$
z=\left[\begin{array}{cc}
A & 0 \\
D & J\left(A^{T}\right)^{-1} J
\end{array}\right] \bmod \widetilde{P}
$$


such that $z \widetilde{P} \in Z_{\widetilde{P}}(\tilde{w})$. Since $X_{B_{G}}(\tilde{w}) \rightarrow X_{\widetilde{P}}(\tilde{w})$ is surjective, we may assume that $z\left(\bmod B_{G}\right) \in X_{B_{G}}(\tilde{w})$, i.e., $z \in \overline{B_{G} \tilde{w} B_{G}}$. Then for every $A^{\prime} \in B_{n}$ :

$$
\left[\begin{array}{cc}
A^{\prime} & 0_{n \times n} \\
0_{n \times n} & J\left(A^{\prime T}\right)^{-1} J
\end{array}\right] z=\left[\begin{array}{cc}
A^{\prime} A & 0 \\
J\left(A^{\prime T}\right)^{-1} J D & J\left(A^{\prime T}\right)^{-1}\left(A^{\prime T}\right)^{-1} J
\end{array}\right]=: z^{\prime} .
$$

Then $z^{\prime} \in \overline{B_{G} \tilde{w} B_{G}}$, so $z^{\prime}(\bmod \widetilde{P}) \in X_{\widetilde{P}}(\tilde{w})$. By Proposition 2.3.4(b), we have that $A$ is invertible, and hence $A A^{\prime}$. This implies again by Proposition 2.3.4(b) that $z^{\prime}(\bmod \widetilde{P}) \in Z_{\widetilde{P}}(\tilde{w})$. Thus $Z_{\widetilde{P}}(\tilde{w})$ is $B_{n}$ stable. Also $p\left(A^{\prime} z\right)=p\left(z^{\prime}\right)=A^{\prime} A=$ $A^{\prime} p(z)$. Hence $p$ is $B_{n}$-equivariant.

Theorem 3.22. With notation as above, let $w^{\prime}:=(k+1, \ldots, r, n, \ldots, r+1, k, \ldots, 1)$ be an element of $S_{n}$, the Weyl group of $\mathrm{GL}_{n}$. Then:

(a) The natural map $X_{\widetilde{P}}(\tilde{w}) \longrightarrow X_{P}(w)$ is proper and birational. In particular, the map $Z_{\widetilde{P}}(\tilde{w}) \longrightarrow Y_{P}(w)$ is proper and birational. And therefore, $Z_{\widetilde{P}}(\tilde{w})$ is a desingularization of $Y_{P}(w)$.

(b) $X_{P_{r-k}^{\prime}}\left(w^{\prime}\right)$ is the fiber of the natural map $Z_{\widetilde{P}}(\tilde{w}) \longrightarrow Y_{P}(w)$ at $e_{\mathrm{id}} \in Y_{P}(w)$.

(c) $X_{P_{r-k}^{\prime}}\left(w^{\prime}\right)$ is the image of $p$. Further, $p$ is a fibration with fiber isomorphic to $V_{w}$.

(d) $p$ identifies $Z_{\widetilde{P}}(\tilde{w})$ as a subbundle of the trivial bundle $O_{G / P}^{-} \times X_{P_{r-k}^{\prime}}\left(w^{\prime}\right)$, which arises as the restriction of the vector bundle on $\mathrm{GL}_{n} / P_{r-k}^{\prime}$ associated to the $P_{r-k}^{\prime}$-module $V_{w}$ (which, in turn, is a $P_{r-k}^{\prime}$-submodule of $O_{G / P}^{-}$).

Proof. (a): The map $X_{\widetilde{P}}(\tilde{w}) \hookrightarrow G / \widetilde{P} \rightarrow G / P$ is proper and its (scheme-theoretic) image is $X_{P}(w)$, hence $X_{\widetilde{P}}(\tilde{w}) \rightarrow X_{P}(w)$ is proper. Birationality follows from the fact that $\tilde{w}$ is the minimal representative of the coset $w \widetilde{P}$.

(b): The fiber at $e_{\text {id }} \in Y_{P}(w)$ of the map $Y_{\widetilde{P}}(\tilde{w}) \longrightarrow Y_{P}(w)$ is $0 \times V_{w}^{\prime}$, inside $V_{w} \times V_{w}^{\prime}=Y_{\widetilde{P}}(\tilde{w})$. Since $Z_{\tilde{P}}(\tilde{w})$ is the closure of $Y_{\widetilde{P}}(\tilde{w})$ inside $O_{G / P}^{-} \times P / \widetilde{P}$ and $X_{P^{\prime}}\left(w^{\prime}\right)$ is the closure of $V_{w}^{\prime}$ inside $P / \widetilde{P}$ (note that as a subvariety of $O_{P / \widetilde{P}}^{-}$, $Y_{P^{\prime}}\left(w^{r-k}\right)^{r}$ is identified with $V_{w}^{\prime}$ ), we see that the fiber at $e_{\text {id }}$ (belonging to $Y_{P}(w)$ ) of ${ }^{r-k} \widetilde{P}_{\tilde{P}}(\tilde{w}) \longrightarrow Y_{P}(w)$ is $X_{P_{r-k}^{\prime}}\left(w^{\prime}\right)$.

(c): From Remark 3.19 we have $p\left(Y_{\widetilde{P}}(\tilde{w})\right)=V_{w}^{\prime} \subseteq X_{P_{r-k}^{\prime}}\left(w^{\prime}\right)$. Since $Y_{\tilde{P}}(\tilde{w})$ is dense inside $Z_{\widetilde{P}}(\tilde{w})$ and $X_{P_{r-k}^{\prime}}\left(w^{\prime}\right)$ is closed in $\operatorname{GL}_{n} / P_{r-k}^{\prime}$ we see that $p\left(Z_{\tilde{P}}(\tilde{w})\right) \subseteq$ $X_{P_{r-k}^{\prime}}\left(w^{\prime}\right)$. The other inclusion $X_{P_{r-k}^{\prime}}\left(w^{\prime}\right) \subseteq p\left(Z_{\tilde{P}}(\tilde{w})\right)$ follows from (b). Hence, $p\left(Z_{\widetilde{P}}^{r-k}(\tilde{w})\right)=X_{P_{r-k}^{\prime}}\left(w^{\prime}\right)$. To prove the second assertion of (c) we shall show that for every $A \in \mathrm{GL}_{n}^{r-k}$ with $A \bmod P_{r-k}^{\prime} \in X_{P_{r-k}^{\prime}}\left(w^{\prime}\right)$, we have that $p^{-1}\left(A \bmod P_{r-k}^{\prime}\right)$ is isomorphic to $V_{w}$.

To prove this we first observe that $p^{-1}\left(e_{\text {id }}\right)$ is isomorphic to $V_{w}$ in view of Remark 3.19. Next observe that every $B_{n}$-orbit inside $X_{P_{r-k}^{\prime}}\left(w^{\prime}\right)$ meets $V_{w}^{\prime}$ (which equals $Y_{P_{r-k}^{\prime}}\left(w^{\prime}\right)$ ); further $p$ is $B_{n}$-equivariant by Proposition 3.21 and hence every fiber is isomorphic to the fiber at $e_{\mathrm{id}}$, i.e., isomorphic to $V_{w}$. 
(d): Define a right action of $\mathrm{GL}_{n}$ on $O_{G / P}^{-}$(identified with $\mathrm{Sym}_{n}$ as in Remark 2.2.6) as $g \circ v=g^{T} v g$ for $g \in \mathrm{GL}_{n}, v \in \mathrm{Sym}_{n}$. This induces an action of $P_{r-k}^{\prime}$ on $O_{G / P}^{-}$ under which $V_{w}$ is stable. Thus we get the homogeneous bundle

$$
\mathrm{GL}_{n} \times{ }^{P_{r-k}^{\prime}} V_{w} \longrightarrow \mathrm{GL}_{n} / P_{r-k}^{\prime} .
$$

Now to prove the assertion about $\left.Z_{\widetilde{P}}(\tilde{w})\right)$ being a vector bundle over $X_{P^{\prime}}\left(w^{\prime}\right)$, we will show that there is a commutative diagram given as below, with $\psi$ an isomorphism:

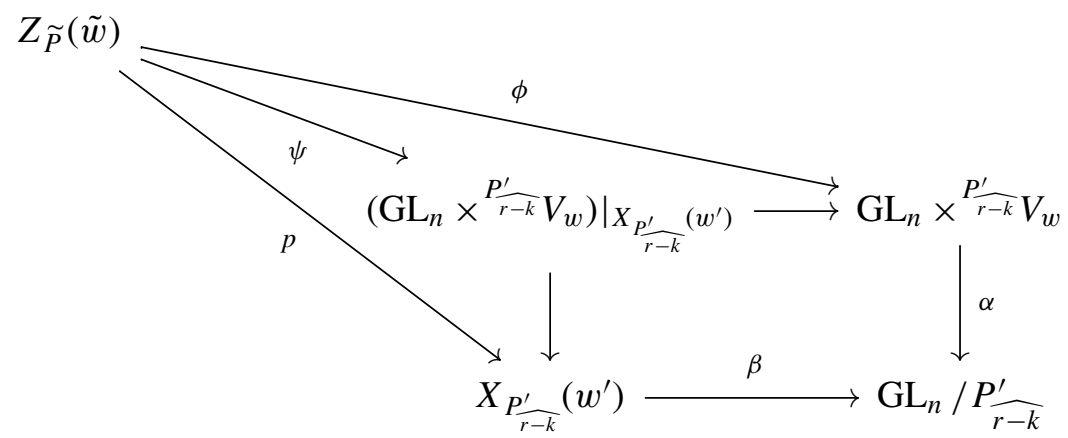

The map $\alpha$ is the homogeneous bundle map and $\beta$ is the inclusion map. Define $\phi$ by

$$
\phi:\left[\begin{array}{cc}
A & 0_{n \times n} \\
D & J\left(A^{T}\right)^{-1} J
\end{array}\right] \bmod \widetilde{P} \longmapsto\left(A, D^{T} J A\right) / \sim .
$$

Using Proposition 2.3.4(c) and Remark 3.19 we conclude the following: $\phi$ is welldefined and injective; $\beta \cdot p=\alpha \cdot \phi$; hence, by the universal property of products, the map $\psi$ exists; and, finally, the injective map $\psi$ is in fact an isomorphism (by dimension considerations).

As an immediate consequence of Theorem 3.22 we have

Corollary 3.23. We have the following realization of Diagram 1.2:

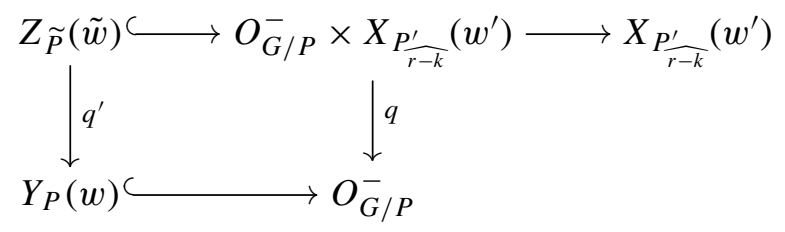

Proposition 3.24. (1) The Schubert variety $X_{P_{r-f}^{\prime}}\left(w^{\prime}\right)$ is isomorphic to the Grassmannian $\mathrm{GL}_{r} / P_{r-k}^{\prime \prime}$, where $P_{r-k}^{\prime \prime}$ is the parabolic subgroup in $\mathrm{GL}_{r}$ obtained by omitting $\alpha_{r-k}$.

(2) $\left.\left.\left(\mathrm{GL}_{n} \times{ }^{P_{r-k}^{\prime}} V_{w}\right)\right|_{X_{P^{\prime}}\left(w^{\prime}\right)} \cong\left(\mathrm{GL}_{n} \times{ }^{P_{r-k}^{\prime}} V_{w}\right)\right|_{\mathrm{GL}_{r} / P_{r-k}^{\prime \prime}} \cong \mathrm{GL}_{r} \times{ }^{P_{r-k}^{\prime \prime}} V_{w}$ as homogeneous vector bundles.

Proof. (1): This is clear. 
(2): Consider the embedding $i: \mathrm{GL}_{r} \hookrightarrow \mathrm{GL}_{n}$ given by

$$
R \mapsto\left[\begin{array}{cc}
R & 0 \\
0 & \mathrm{Id}_{n-r}
\end{array}\right]
$$

Define the action of $\mathrm{GL}_{r}$ on $\mathrm{Sym}_{n}$ as the action induced by this embedding. This induces an action of $P_{r-k}^{\prime \prime}$ on $\operatorname{Sym}_{n}$. As $i\left(P_{r-k}^{\prime \prime}\right) \subset P_{r-k}^{\prime}$, the $P_{r-k}^{\prime}$ stability of $V_{w}$ implies the $P_{r-k}^{\prime \prime}$ stability of $V_{w}$. Hence our result follows.

Corollary 3.25. We have the following realization of Diagram 1.2:

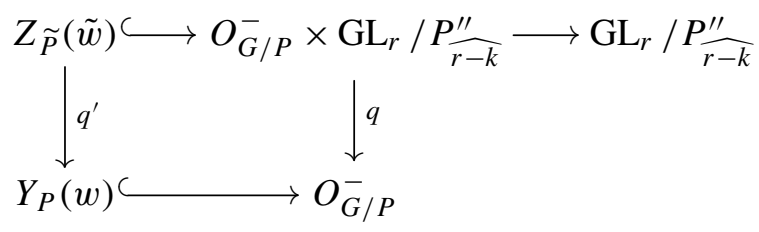

\section{Free resolutions}

Kempf-Lascoux-Weyman geometric technique. We summarize the geometric technique of computing free resolutions, following [Weyman 2003, Chapter 5].

Consider Diagram 1.1. There is a natural map $f: V \longrightarrow$ Grass $_{r, d}$ (where $r=\operatorname{rk}_{V} Z$ and $d=\operatorname{dim} \mathbb{A}$ ) such that the inclusion $Z \subseteq \mathbb{A} \times V$ is the pull-back of the tautological sequence (2.4.4); here $\operatorname{rk}_{V} Z$ denotes the rank of $Z$ as a vector bundle over $V$, i.e., $\operatorname{rk}_{V} Z=\operatorname{dim} Z-\operatorname{dim} V$. Let $\xi=\left(f^{*} \mathcal{Q}\right)^{*}$. Write $R$ for the polynomial ring $\mathbb{C}[\mathbb{A}]$ and $\mathfrak{m}$ for its homogeneous maximal ideal. (The grading on $R$ arises as follows. In Diagram 1.1, $\mathbb{A}$ is thought of as the fiber of a trivial vector bundle, so it has a distinguished point, its origin. Now, being a subbundle, $Z$ is defined by linear equations in each fiber; i.e., for each $v \in V$, there exist $s:=\left(\operatorname{dim} \mathbb{A}-\operatorname{rk}_{V} Z\right)$ linearly independent linear polynomials $\ell_{v, 1}, \ldots, \ell_{v, s}$ that vanish along $Z$ and define it. Now $Y=\left\{y \in \mathbb{A}\right.$ : there exists $v \in V$ such that $\left.\ell_{v, 1}(y)=\cdots=\ell_{v, s}(y)=0\right\}$. Hence $Y$ is defined by homogeneous polynomials. This explains why the resolution obtained below is graded.) Let $\mathfrak{m}$ be the homogeneous maximal ideal, i.e., the ideal defining the origin in $\mathbb{A}$. Then:

Theorem 4.1 [Weyman 2003, Basic Theorem 5.1.2]. With notation as above, there is a finite complex $\left(F_{.}, \partial_{\bullet}\right)$ of finitely generated graded free $R$-modules that is quasi-isomorphic to $\mathbf{R} q_{*}^{\prime} \mathrm{O}_{Z}$, with

$$
F_{i}=\bigoplus_{j \geq 0} H^{j}\left(V, \bigwedge^{i+j} \xi\right) \otimes_{\mathbb{C}} R(-i-j),
$$

and $\partial_{i}\left(F_{i}\right) \subseteq \mathfrak{m} F_{i-1}$. Furthermore, the following are equivalent:

(a) $Y$ has rational singularities i.e., $\mathbf{R} q_{*}^{\prime} \mathrm{O}_{Z}$ is quasi-isomorphic to $\mathrm{O}_{Y}$;

(b) $F_{\text {. }}$ is a minimal $R$-free resolution of $\mathbb{C}[Y]$, i.e., $F_{0} \simeq R$ and $F_{-i}=0$ for every $i>0$. 
A sketch of the proof is given in [Kummini et al. 2015, Section 4], and [Weyman 2003, 5.1.3] may be consulted for a more comprehensive account.

Our situation. We now apply Theorem 4.1 to our situation. We keep the notation of Theorem 3.22. Theorem 4.1 and Corollary 3.25 yield the following result:

Theorem 4.2. Write $\xi$ for the homogeneous vector bundle on $\mathrm{GL}_{r} / P_{r-k}^{\prime \prime}$ associated to the $P_{r-k}^{\prime \prime}$-module $\left(O_{G / P}^{-} / V_{w}\right)^{*}$ (this is the dual of the quotient of $O_{G / P}^{-} \times$ $\mathrm{GL}_{r} / P_{r-k}^{\prime \prime}$ by $\left.Z_{\widetilde{P}}(\tilde{w})\right)$. Then we have a minimal $R$-free resolution $\left(F_{\bullet}, \partial_{\bullet}\right)$ of $\mathbb{C}\left[Y_{P}(w)\right]$ with

$$
F_{i}=\bigoplus_{j \geq 0} H^{j}\left(\mathrm{GL}_{r} / P_{r-k}^{\prime \prime}, \bigwedge^{i+j} \xi\right) \otimes_{\mathbb{C}} R(-i-j) .
$$

Computing the cohomology groups required in Theorem 4.2 in the general situation is a difficult problem. Techniques for computing them in our specific case are discussed in the following section.

\section{Cohomology of homogeneous vector bundles}

We have shown in Theorem 4.2 that the calculation of a minimal $R$-free resolution of $\mathbb{C}\left[Y_{P}(w)\right]$ comes down to the computation of the cohomology of certain homogeneous bundles over $\mathrm{GL}_{r} / P_{r-k}^{\prime \prime}$. In particular we need to calculate

$$
H^{\bullet}\left(\mathrm{GL}_{r} / P_{r-k}^{\prime \prime}, \bigwedge^{t} \xi\right)
$$

for arbitrary $t$.

The $P_{r-k}^{\prime \prime}$-module $\left(O_{G / P}^{-} / V_{w}\right)^{*}$ is not completely reducible (the unipotent radical

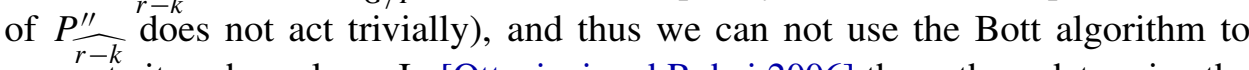
compute its cohomology. In [Ottaviani and Rubei 2006] the authors determine the cohomology of general homogeneous bundles on Hermitian symmetric spaces. As $\mathrm{GL}_{r} / P_{r-k}^{\prime \prime}$ is such a space their results could be used to determine (5.1). In practice, proceeding along these lines is possible though extremely complicated.

Another approach to the calculation of these cohomologies comes from using a technique employed in [Weyman 2003, Chapter 6.3]. There the minimal $R$-free resolution of a related space is computed and the minimal $R$-free resolution of $\mathbb{C}\left[Y_{P}(w)\right]$ can be seen as a subresolution. In [Weyman 2003] this method is used for the case when $n=r$. That is, the case where $Y_{P}(w)$ is the symmetric determinental variety. In this case the authors assume that $k=2 u$ (the odd case can be reduced to this even case). They look at the subspace $T_{w}$ of $\mathrm{Sym}_{n}$ given by symmetric matrices of block form

$$
\left[\begin{array}{cc}
0_{n-u \times n-u} & R \\
R^{T} & S_{u \times u}
\end{array}\right]
$$


Let $P_{\widehat{n-u}}^{\prime}$ be the parabolic subgroup of $\mathrm{GL}_{n}$ omitting the root $\alpha_{n-u}$, then $T_{w}$ is a $P_{\widehat{n-u}}^{\prime}$-module under the same action. If $Z_{w}$ is the homogeneous vector bundle associated with $T_{w}$ we have the following diagram

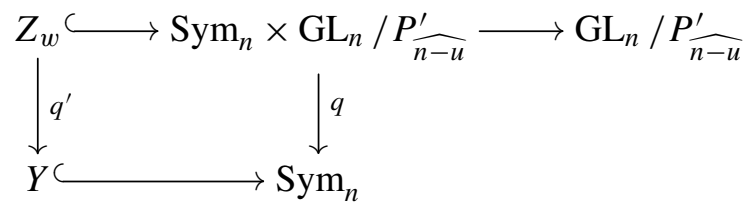

They show that the resolution of $\mathbb{C}\left[Y_{P}(w)\right]$ can be realized as a subresolution of the resolution of $\mathbb{C}[Y]$. In this case, the $P_{n-u}^{\prime}$-module $\left(\operatorname{Sym}_{n} / T_{w}\right)^{*}$ (this is the dual of the quotient of $\operatorname{Sym}_{n} \times \mathrm{GL}_{n} / P_{n-u}^{\prime}$ by $Z_{w}$ ) is completely reducible and thus the cohomology of the corresponding homogeneous vector bundles $\bigwedge^{t} \xi$ may be computed using the Bott algorithm, leading to this:

Theorem 5.2 [Weyman 2003, Theorem 6.3.1(c)]. The $i$-th term $G_{i}$ of the minimal free resolution of $\mathbb{C}\left[Y_{P}(w)\right]$ as an $R$ module is given by the formula

$$
G_{i}=\bigoplus_{\substack{\lambda \in Q_{k-1}(2 t) \\ \text { rank } \lambda \text { even } \\ i=t-k \frac{1}{2} \text { rank } \lambda}} S_{\lambda} \vee \mathbb{C}^{n} \otimes_{\mathbb{C}} R .
$$

Here $Q_{k-1}(2 t)$ is the set of partitions $\lambda$ of $2 t$ which in hook notation can be written as $\lambda=\left(a_{1}, \ldots, a_{s} \mid b_{1}, \ldots, b_{s}\right)$, where $s$ is a positive integer, and for each $j$ we have $a_{j}=b_{j}+(k-1)$. And $\lambda^{\vee}$ is the conjugate (or dual) partition of $\lambda$. And finally, rank $\lambda$ is defined as being equal to $l$, where the largest square fitting inside $\lambda$ is of size $l \times l$.

Similar methods may be used to compute a closed form formula for the minimal free resolution of $\mathbb{C}\left[Y_{P}(w)\right]$ as an $R$ module in the case $r \neq n$.

\section{References}

[Billey and Lakshmibai 2000] S. Billey and V. Lakshmibai, Singular loci of Schubert varieties, Progress in Mathematics 182, Birkhäuser, Boston, 2000. MR 2001j:14065 Zbl 0959.14032

[Borel 1991] A. Borel, Linear algebraic groups, 2nd ed., Graduate Texts in Mathematics 126, Springer, New York, 1991. MR 92d:20001 Zbl 0726.20030

[Bourbaki 1968] N. Bourbaki, "Éléments de mathématique, XXXIV: Groupes et algèbres de Lie, chapitres 4 à 6", pp. 288 Actualités Scientifiques et Industrielles 1337, Hermann, Paris, 1968. MR 39 \#1590 Zbl 0186.33001

[Edixhoven 1992] B. Edixhoven, "Néron models and tame ramification", Compositio Math. 81:3 (1992), 291-306. MR 93a:14041 Zbl 0759.14033

[Fulton and Harris 1991] W. Fulton and J. Harris, Representation theory: A first course, Graduate Texts in Mathematics 129, Springer, New York, 1991. MR 93a:20069 Zbl 0744.22001 
[Jantzen 2003] J. C. Jantzen, Representations of algebraic groups, 2nd ed., Mathematical Surveys and Monographs 107, Amer. Math. Soc., Providence, RI, 2003. MR 2004h:20061 Zbl 1034.20041

[Józefiak et al. 1981] T. Józefiak, P. Pragacz, and J. Weyman, "Resolutions of determinantal varieties and tensor complexes associated with symmetric and antisymmetric matrices", pp. 109-189 in Young tableaux and Schur functors in algebra and geometry (Torun, 1980), Astérisque 87, Société Mathématique de France, Paris, 1981. MR 83j:14044 Zbl 0488.14012

[Kummini et al. 2015] M. Kummini, V. Lakshmibai, S. Pramathanath, and C. S. Seshadri, "Free resolutions of some Schubert singularities", preprint, 2015. arXiv 1504.04415

[Lakshmibai 1987] V. Lakshmibai, "Geometry of $G / P$, VII: The symplectic group and the involution $\sigma ”$, J. Algebra 108:2 (1987), 403-434. MR 88i:14046b Zbl 0618.14027

[Lakshmibai and Raghavan 2008] V. Lakshmibai and K. N. Raghavan, Standard monomial theory: Invariant theoretic approach, Encyclopaedia of Mathematical Sciences 137, Springer, Berlin, 2008. MR 2008m:14095 Zbl 1137.14036

[Lascoux 1978] A. Lascoux, "Syzygies des variétés déterminantales", Adv. in Math. 30:3 (1978), 202-237. MR 80j:14043 Zbl 0394.14022

[Ottaviani 1995] G. Ottaviani, "Rational homogeneous varieties", notes from SMI course in Algebraic Geometry, Cortona, Italy, 1995, http://tinyurl.com/ottavianiRHV95.

[Ottaviani and Rubei 2006] G. Ottaviani and E. Rubei, "Quivers and the cohomology of homogeneous vector bundles”, Duke Math. J. 132:3 (2006), 459-508. MR 2008b:14075 Zbl 1100.14012

[Seshadri 2007] C. S. Seshadri, Introduction to the theory of standard monomials, Texts and Readings in Mathematics 46, Hindustan Book Agency, New Delhi, 2007. MR 2008m:14099 Zbl 1133.14052

[Snow 1994] D. M. Snow, "Homogeneous vector bundles", preprint, 1994, http://www3.nd.edu/ $\sim$ snow/Papers/HomogVB.pdf.

[Steinberg 1968] R. Steinberg, "Lectures on Chevalley groups", Lecture notes, Yale University, 1968, https://math.depaul.edu/cdrupies/research/papers/chevalleygroups.pdf.

[Weyman 2003] J. Weyman, Cohomology of vector bundles and syzygies, Cambridge Tracts in Mathematics 149, Cambridge Univ. Press, 2003. MR 2004d:13020 Zbl 1075.13007

Received May 28, 2015. Revised August 27, 2015.

VENKATRAMANI LAKSHMIBAI

MATHEMATICS DEPARTMENT

567 LAKE HALL

NORTHEASTERN UNIVERSITY

BOSTON, MA 02115

UNITED STATES

v.lakshmibai@neu.edu

ReUven Hodges

MATHEMATICS Department

567 LAKE HALL

NORTHEASTERN UNIVERSITY

BOSTON, MA 02115

UNITED STATES

hodges.r@husky.neu.edu 


\title{
PACIFIC JOURNAL OF MATHEMATICS
}

\author{
msp.org/pjm
}

Founded in 1951 by E. F. Beckenbach (1906-1982) and F. Wolf (1904-1989)

\section{EDITORS}

Don Blasius (Managing Editor)

Department of Mathematics

University of California

Los Angeles, CA 90095-1555

blasius@math.ucla.edu

\author{
Paul Balmer \\ Department of Mathematics \\ University of California \\ Los Angeles, CA 90095-1555 \\ balmer@math.ucla.edu \\ Robert Finn \\ Department of Mathematics \\ Stanford University \\ Stanford, CA 94305-2125 \\ finn@math.stanford.edu \\ Sorin Popa \\ Department of Mathematics \\ University of California \\ Los Angeles, CA 90095-1555 \\ popa@math.ucla.edu
}

\author{
Vyjayanthi Chari \\ Department of Mathematics \\ University of California \\ Riverside, CA 92521-0135 \\ chari@math.ucr.edu \\ Kefeng Liu \\ Department of Mathematics \\ University of California \\ Los Angeles, CA 90095-1555 \\ liu@math.ucla.edu \\ Jie Qing \\ Department of Mathematics \\ University of California \\ Santa Cruz, CA 95064 \\ qing@ cats.ucsc.edu
}

\section{PRODUCTION}

Silvio Levy, Scientific Editor, production@msp.org

\section{SUPPORTING INSTITUTIONS}

ACADEMIA SINICA, TAIPEI

CALIFORNIA INST. OF TECHNOLOGY

INST. DE MATEMÁTICA PURA E APLICADA

KEIO UNIVERSITY

MATH. SCIENCES RESEARCH INSTITUTE

NEW MEXICO STATE UNIV.

OREGON STATE UNIV.

\author{
STANFORD UNIVERSITY \\ UNIV. OF BRITISH COLUMBIA \\ UNIV. OF CALIFORNIA, BERKELEY \\ UNIV. OF CALIFORNIA, DAVIS \\ UNIV. OF CALIFORNIA, LOS ANGELES \\ UNIV. OF CALIFORNIA, RIVERSIDE \\ UNIV. OF CALIFORNIA, SAN DIEGO \\ UNIV. OF CALIF., SANTA BARBARA
}

\author{
Daryl Cooper \\ Department of Mathematics \\ University of California \\ Santa Barbara, CA 93106-3080 \\ cooper@math.ucsb.edu \\ Jiang-Hua Lu \\ Department of Mathematics \\ The University of Hong Kong \\ Pokfulam Rd., Hong Kong \\ jhlu@maths.hku.hk \\ Paul Yang \\ Department of Mathematics \\ Princeton University \\ Princeton NJ 08544-1000 \\ yang@math.princeton.edu
}

These supporting institutions contribute to the cost of publication of this Journal, but they are not owners or publishers and have no responsibility for its contents or policies.

See inside back cover or msp.org/pjm for submission instructions.

The subscription price for 2015 is US \$420/year for the electronic version, and \$570/year for print and electronic.

Subscriptions, requests for back issues and changes of subscribers address should be sent to Pacific Journal of Mathematics, P.O. Box 4163, Berkeley, CA 94704-0163, U.S.A. The Pacific Journal of Mathematics is indexed by Mathematical Reviews, Zentralblatt MATH, PASCAL CNRS Index, Referativnyi Zhurnal, Current Mathematical Publications and Web of Knowledge (Science Citation Index).

The Pacific Journal of Mathematics (ISSN 0030-8730) at the University of California, c/o Department of Mathematics, 798 Evans Hall \#3840, Berkeley, CA 94720-3840, is published twelve times a year. Periodical rate postage paid at Berkeley, CA 94704, and additional mailing offices. POSTMASTER: send address changes to Pacific Journal of Mathematics, P.O. Box 4163, Berkeley, CA 94704-0163.

PJM peer review and production are managed by EditFLOW ${ }^{\circledR}$ from Mathematical Sciences Publishers.

\section{PUBLISHED BY}

\section{mathematical sciences publishers \\ nonprofit scientific publishing}

http://msp.org/

(C) 2015 Mathematical Sciences Publishers 


\title{
PACIFIC JOURNAL OF MATHEMATICS
}

\author{
Volume 279 No. 1-2 December 2015
}

In memoriam: Robert Steinberg

Robert Steinberg (1922-2014): In memoriam V. S. VARADARAJAN

Cellularity of certain quantum endomorphism algebras

HENNING H. ANDERSEN, GUSTAV I. LEHRER and RUIBIN ZHANG

Lower bounds for essential dimensions in characteristic 2 via orthogonal representations ANTONIO BABIC and VLADIMIR CHERNOUSOV

Cocharacter-closure and spherical buildings

Michael Bate, Sebastian Herpel, Benjamin Martin and Gerhard RöHrLe

Embedding functor for classical groups and Brauer-Manin obstruction

Eva Bayer-Fluckiger, Ting-Yu LeE and Raman Parimala

On maximal tori of algebraic groups of type $G_{2}$

Constantin Beli, PhilipPe Gille and Ting-Yu LeE

On extensions of algebraic groups with finite quotient

MICHEL BRION

Essential dimension and error-correcting codes

SHANE CERNELE and ZiNOVy REICHSTEIN

Notes on the structure constants of Hecke algebras of induced representations of finite Chevalley groups

Charles W. CuRTis

Complements on disconnected reductive groups

FRANÇOIS DIGNE and JEAN MICHEL

Extending Hecke endomorphism algebras

Jie Du, Brian J. Parshall and LeOnard L. SCOTT

Products of partial normal subgroups

ELLEN HENKE

Lusztig induction and $\ell$-blocks of finite reductive groups

RADHA KESSAR and GUNTER MALLE

Free resolutions of some Schubert singularities

Manoj Kummini, Venkatramani Lakshmibai, Pramathanath Sastry and C. S. Seshadri

Free resolutions of some Schubert singularities in the Lagrangian Grassmannian

VenKatramani LAKSHMibai and ReUVEN HODGES

Distinguished unipotent elements and multiplicity-free subgroups of simple algebraic groups

Martin W. Liebeck, Gary M. Seitz and Donna M. Testerman

Action of longest element on a Hecke algebra cell module

GEORGE LUSZTIG

Generic stabilisers for actions of reductive groups

BENJAMIN MARTIN

On the equations defining affine algebraic groups

VLADIMIR L. POPOV

Smooth representations and Hecke modules in characteristic $p$

PETER SCHNEIDER

On CRDAHA and finite general linear and unitary groups

BHAMA SRINIVASAN

Weil representations of finite general linear groups and finite special linear groups PHAM HUU TIEP

The pro- $p$ Iwahori Hecke algebra of a reductive $p$-adic group, $\mathrm{V}$ (parabolic induction) MARIE-FRANCE VIGNÉRAS

Acknowledgement 